\title{
On the Presentations of Extended Affine Weyl Groups
}

\author{
By
}

Saeid Azam* and Valiollah Shahsanaei*

\begin{abstract}
We give a finite presentation for reduced non-simply laced extended affine Weyl groups of arbitrary nullity. When nullity is less than or equal to 3 , this presentation reduces to a very simple presentation in which the generators and relations can be easily read from a set of data attached to the root system.
\end{abstract}

\section{$\S 0 . \quad$ Introduction}

Groups generated by reflections play an important role in many fields of mathematics. In Lie theory the Weyl groups of Lie algebras with root space decompositions are groups generated by reflections. Different Weyl groups possess different features which depend on the nature of the corresponding Lie algebra or root system. In recent years the fantastic nature of extended affine Weyl groups has captured interests of many people. It is now revealed that this is mostly because of an intrinsic normal subgroup, called the Heisenberg-like group, which has a very reach internal structure. The study of such Weyl groups is therefore interesting both in terms of the application and also from the group theory point of view. In this work we study the existence of finite presentations for extended affine Weyl groups and their Heisenberg-like counterparts.

\footnotetext{
Communicated by K. Saito. Received December 1, 2006. Revised August 10, 2007.

2000 Mathematics Subject Classification(s): 20F55; Secondary: 17B67.

This research was in part supported by a grant from Institute for Studies in Theoretical Physics and Mathematics (IPM) (No. 85170046).

*The Center for Excellence in Mathematics, University of Isfahan, Isfahan, P.O.Box 81745163 , Iran.

e-mail: azam@sci.ui.ir.

** University of Isfahan, Isfahan, P.O.Box 81745-163, Iran.

e-mail: sanaei@math.ui.ac.ir.
}

(c) 2008 Research Institute for Mathematical Sciences, Kyoto University. All rights reserved. 
Let $R$ be a reduced non-simply laced extended affine root system of rank $\ell$, nullity $\nu$ and twist number $t$, with extended affine Weyl group $\mathcal{W}$ (see Definition 2.1). As a byproduct of our main theorem (Theorem 3.1), we prove that if $\nu \leq 3$, then $\mathcal{W}$ has a finite presentation defined by generators

$$
\left\{\begin{array}{l}
\hat{w}_{i}, \hat{t}_{i, r}, 1 \leq i \leq \ell, 1 \leq r \leq \nu \\
\hat{z}_{r, s}, \quad 1 \leq r<s \leq \nu
\end{array}\right.
$$

and relations

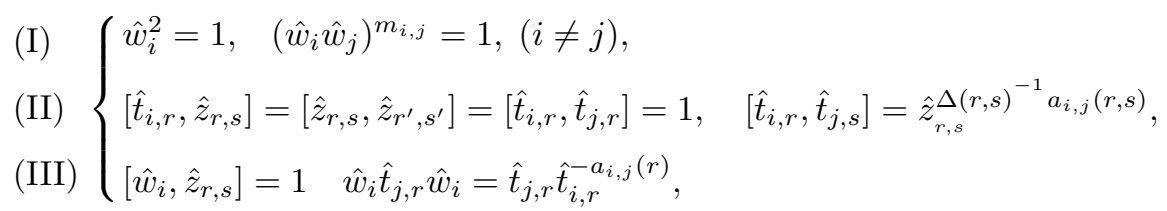

where $m_{i, j}, a_{i, j}(r), a_{i, j}(r, s)$ and $\Delta(r, s)$ are integers introduced by (3.2), (2.16), (2.17) and (2.12), respectively (see Corollaries 3.1, 3.2 and 3.3). Considering the semidirect product $\mathcal{W}=\dot{\mathcal{W}} \ltimes \mathcal{H}$ (Proposition 2.1), we may interpret the above presentation as follows. The generators $\hat{w}_{i}$ together with relations of the form (I) afford the Coxeter presentation for the finite Weyl group $\dot{\mathcal{W}}$. The generators $\hat{t}_{i, r}$ and $\hat{z}_{r, s}$ together with relations of the form (II) present the Heisenberg-like group $\mathcal{H}$, and finally the relations of the form (III) are imposed by the semidirect product interaction between $\dot{\mathcal{W}}$ and $\mathcal{H}$. The integers $m_{i, j}$ can be read from the finite Cartan matrix, and the integers $a_{i, j}(r), a_{i, j}(r, s)$ and $\Delta(r, s)$ are easily computed using certain root data. The presentation given above for nullity $\leq 3$ is in fact a consequence of our general finite presentation given for arbitrary nullity (Theorem 3.1).

We emphasize that the only known finite presentation for extended affine Weyl groups of nullity $>1$ is the so called generalized Coxeter presentation ([ST]) which is given only for nullity 2. Therefore for $\nu>2$ our result gives, for the first time, a finite presentation for reduced non-simply laced extended affine Weyl groups. For simply laced extended affine Weyl groups, a presentation with similar nature is given in [AzS2] and it is used to determine the existence of the so called a presentation by conjugation for the corresponding Weyl groups $([\mathrm{AzS} 1])$. In an under going project, using the results of the present work, we investigate the existence of presentation by conjugation for the Weyl groups under consideration (see also $[\mathrm{H}]$ and $[\mathrm{Az} 2]$ ).

The paper is arranged as follows. Section 1 contains the basic concepts about semilattices and extended affine root systems. Section 2 forms the core of the paper and contains several important results about the structure of an 
extended affine Weyl group and its Heisenberg-like subgroup (see for example Proposition 2.1). Our main results about the presentations of extended affine Weyl groups appear in Section 3 (see Theorem 3.1 and its corollaries). The paper is concluded with a section of examples, Section 4 . The reader would find it helpful in understanding the notations and terms appearing throughout the paper if he or she refers to Section 4 time to time while reading the paper.

For the study of extended affine root systems and their Weyl groups we refer the reader to $[\mathrm{Sa}],[\mathrm{MS}],[\mathrm{AABGP}],[\mathrm{AzS} 2],[\mathrm{AzS} 1],[\mathrm{Az} 1],[\mathrm{Az} 2],[\mathrm{SaT}]$, $[\mathrm{Az} 4],[\mathrm{T} 2]$ and $[\mathrm{T} 1]$.

\section{$\S 1$. Preliminaries}

In this section, we briefly recall the definition of supporting class of a semilattice from [AzS2]. Also we record some basic properties of the structure of reduced non-simply laced extended affine root systems. For the theory of extended affine root systems the reader is referred to [AABGP]. In particular, we will use the notation and concepts introduced there without further explanations.

Let $S$ be a semilattice of rank $\nu$ in a real vector space $\mathcal{V}^{0}$. This means that $\mathcal{V}^{0}$ is a $\nu$-dimensional real vector space and $S$ is a subset of $\mathcal{V}^{0}$ satisfying

$$
0 \in S, \quad S \pm 2 S \subset S, \quad S \text { is discrete in } \mathcal{V}^{0}, \quad \text { and } S \text { spans } \mathcal{V}^{0} .
$$

A subset $\mathcal{B}$ of $S$ is said to be a basis for $S$, if it is a $\mathbb{Z}$-basis for the $\mathbb{Z}$-span $\langle S\rangle$ of $S$. Then from [AABGP, II.1.11] and [AzS2, §2], it follows that $S$ has a basis $\mathcal{B}=\left\{\sigma_{1}, \ldots, \sigma_{\nu}\right\}$ and there is a unique set, denoted $\operatorname{supp}_{\mathcal{B}}(S)$, consisting of subsets of $\{1, \ldots, \nu\}$ such that

$$
\emptyset \in \operatorname{supp}_{\mathcal{B}}(S) \quad \text { and } \quad S=\bigcup_{J \in \operatorname{Supp}_{\mathcal{B}}(S)}\left(\sum_{r \in J} \sigma_{r}+2\langle S\rangle\right),
$$

(If $J=\emptyset$ we set by convention $\sum_{j \in J} \sigma_{j}=0$ ). Since $\mathcal{B} \subseteq S$, we have $\{r\} \in \operatorname{supp}_{\mathcal{B}}(S)$ for all $1 \leq r \leq \nu$. The collection $\operatorname{supp}_{\mathcal{B}}(S)$ is called the supporting class of $S$ (with respect to the basis $\mathcal{B}$ ). We call the integer ind $(S):=$ $\left|\operatorname{supp}_{\mathcal{B}}(S)\right|-1$, index of $S$. Finally, the supporting function of $S$ relative to $\mathcal{B}$, is defined by

$$
\delta:\{1, \ldots, \nu\} \times\{1, \ldots, \nu\} \longrightarrow\{ \pm 1, \pm 2\}
$$




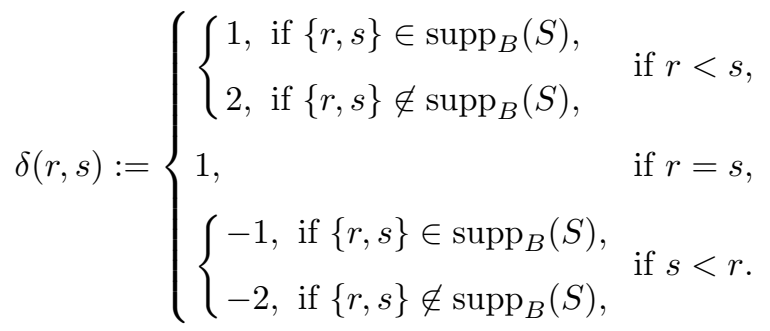

Clearly, if $S$ is a lattice, then $\delta(s, r)=-1$ and $\delta(r, s)=1$ for all $1 \leq r<s \leq \nu$.

The subset

(1.3) $\mathcal{J}_{\mathcal{B}}(S):=\left\{J \in \operatorname{supp}_{\mathcal{B}}(S) \mid\{r, s\} \notin \operatorname{supp}_{\mathcal{B}}(S)\right.$ for some $\left.r, s \in J\right\}$

of $\operatorname{supp}_{\mathcal{B}}(S)$ will appear frequently in our work. Clearly we have

$$
|J| \geq 3 \text { for all } J \in \mathcal{J}_{\mathcal{B}}(S) \text { and }\left|\mathcal{J}_{\mathcal{B}}(S)\right| \leq 2^{\nu}-\nu-\left(\begin{array}{l}
\nu \\
2
\end{array}\right)-1 .
$$

Lemma 1.1. (i) If $\{r, s\} \in \operatorname{supp}_{\mathcal{B}}(S)$ for all $1 \leq r<s \leq \nu$ (in particular, if $S$ is a lattice) or $\operatorname{rank}(S) \leq 2$, then $\mathcal{J}_{\mathcal{B}}(S)=\emptyset$.

(ii) If $\operatorname{rank}(S) \leq 3$, then $\left|\mathcal{J}_{\mathcal{B}}(S)\right| \leq 1$.

(iii) Up to similarity, the semilattices $S$ of rank $\nu \leq 3$ in $\Lambda$ with the basis $\mathcal{B}$ are listed in the following table according to their supporting classes:

Table 1.4. The supporting classes of $S$, up to similarity, for rank $\nu \leq 3$.

\begin{tabular}{c|c|c}
$\operatorname{rank}(S)$ & ind $(S)$ & $\operatorname{supp}_{\mathcal{B}}(S)$ \\
\hline 0 & 0 & $\{\emptyset\}$ \\
\hline 1 & 1 & $\{\emptyset,\{1\}\}$ \\
\hline 2 & 2 & $\{\emptyset,\{1\},\{2\},\{1,2\}\}$ \\
\hline 3 & 3 & $\{\emptyset,\{1\},\{2\},\{3\}\}$ \\
& 3 & $\{\emptyset,\{1\},\{2\},\{3\},\{2,3\}\}$ \\
& 4 & $\{\emptyset,\{1\},\{2\},\{3\},\{1,3\},\{2,3\}\}$ \\
& 5 & $\{\emptyset,\{1\},\{2\},\{3\},\{1,2\},\{1,3\},\{2,3\}\}$ \\
& 6 & $\{\emptyset,\{1\},\{2\},\{3\},\{1,2\},\{1,3\},\{2,3\},\{1,2,3\}\}$ \\
\hline
\end{tabular}

Proof. Parts (i) and (ii) immediately follow from the way $\mathcal{J}_{\mathcal{B}}(S)$ is defined. Also from [AABGP, Table II.4.5], it follows that (iii) holds. 
Here we recall the definition of an extended affine root system from [AABGP, II.2.1]. (In general for a subset $A$ of a vector space equipped with a symmetric bilinear form we set $A^{\times}=\{\alpha \in A \mid(\alpha, \alpha) \neq 0\}$ and $A^{0}=\{\alpha \in A \mid$ $(\alpha, \alpha)=0\}$.

Definition 1.1. A subset $R$ of a non-trivial finite dimensional real vector space $\mathcal{V}$, equipped with a positive semi-definite symmetric bilinear form $(\cdot, \cdot)$, is called an extended affine root system if $R$ satisfies the following 8 axioms:

- R1) $0 \in R$,

- $\mathrm{R} 2)-R=R$,

- R3) $R$ spans $\mathcal{\nu}$,

- R4) $\alpha \in R^{\times} \Longrightarrow 2 \alpha \notin R$,

- R5) $R$ is discrete in $\mathcal{V}$,

- R6) For $\alpha \in R^{\times}$and $\beta \in R$, there exist non-negative integers $d$, $u$ such that $\beta+n \alpha \in R, n \in \mathbb{Z}$, if and only if $-d \leq n \leq u$, moreover $\left(\beta, \alpha^{\vee}\right)=d-u$,

- R7) If $R^{\times}=R_{1} \cup R_{2}$, where $\left(R_{1}, R_{2}\right)=0$, then either $R_{1}=\emptyset$ or $R_{2}=\emptyset$,

- R8) For any $\sigma \in R^{0}$, there exists $\alpha \in R^{\times}$such that $\alpha+\sigma \in R$.

The dimension $\nu$ of the radical $\nu^{0}$ of the form is called the nullity of $R$, and the dimension $\ell$ of $\overline{\mathcal{V}}:=\mathcal{V} / \mathcal{V}^{0}$ is called the rank of $R$.

According to [AABGP, II.2.9], the set $\bar{R}$, the image of $R$ under the canonical map ${ }^{-}: \mathcal{V} \rightarrow \overline{\mathcal{V}}$ is an irreducible finite root system in $\overline{\mathcal{V}}$. The type of $R$ is defined to be the type of $\bar{R}$. The extended affine root system $R$ is called simply laced if the finite root system $\bar{R}$ is simply laced, that is it has one of the types $A_{\ell}(\ell \geq 1), D_{\ell}(\ell \geq 4)$ or $E_{\ell}(\ell=6,7,8)$. The extended affine root system $R$ is called reduced if the finite root system $\bar{R}$ is reduced, that is it has one of the types $A_{\ell}(\ell \geq 1), B_{\ell}(\ell \geq 2), C_{\ell}(\ell \geq 3), D_{\ell}(\ell \geq 4), E_{\ell}(\ell=6,7,8), F_{4}$ or $G_{2}$, and not the one of type $B C_{\ell}(\ell \geq 1)$. In this work we assume that $R$ is a reduced nonsimply laced extended affine root system in $\mathcal{V}$ of rank $\ell$ and nullity $\nu$ relative to the form $(\cdot, \cdot)$.

Remark 1. The notion of a "reduced" extended affine root system which we have used above is defined in [AABGP] and it is different from the notion 
of a "reduced marked" extended affine root system appearing in [Sa]. In [Az3], the author clarifies the relation between extended affine root systems of Saito and those from [AABGP]. In particular in nullity 2 the classification of marked elliptic root systems of Saito [Sa] and extended affine root systems of [AABGP] is compared. Also the notion of marking is analyzed in nullity 3. In general the notion of marking decreases the number of possible root systems appearing in the classification. (See [Az3, Remarks 11-13]).

By [AABGP, II.2.37], We may fix a complement $\dot{\mathcal{V}}$ of $\mathcal{V}^{0}$ in $\mathcal{V}$ such that

$$
\dot{R}:=\left\{\dot{\alpha} \in \dot{\mathcal{V}} \mid \dot{\alpha}+\sigma \in R \text { for some } \sigma \in \mathcal{V}^{0}\right\}
$$

is a finite root system of type $X_{\ell}$ in $\dot{\mathcal{V}}$. Then we can write $\dot{R} \backslash\{0\}=\dot{R}_{s h} \cup \dot{R}_{l g}$ where $\dot{R}_{s h}$ and $\dot{R}_{l g}$ are the sets of short and long roots of $\dot{R}$, respectively. Let

$$
S:=\left\{\sigma \in \mathcal{V}^{0} \mid \alpha+\sigma \in R, \text { for some } \alpha \in \dot{R}_{s h}\right\},
$$

and

$$
L:=\left\{\sigma \in \mathcal{V}^{0} \mid \alpha+\sigma \in R, \text { for some } \alpha \in \dot{R}_{l g}\right\} .
$$

Then $S$ and $L$ are semilattices in $\mathcal{V}^{0}$. Set

$$
k= \begin{cases}2, & \text { if } X_{\ell}=B_{\ell}, C_{\ell} \text { or } F_{4}, \\ 3 & \text { if } X_{\ell}=G_{2} .\end{cases}
$$

From [AABGP, II.4.11] we have $|\langle S\rangle /\langle L\rangle|=k^{t}$ for some integer $1 \leq t \leq \nu$. The integer $t$ is called twist number of $R$. By [AABGP, Lemma 4.15 and Proposition 4.17] there are subspaces $\mathcal{V}_{1}^{0}$ and $\mathcal{V}_{2}^{0}$ of $\mathcal{V}^{0}$ of dimensions $t$ and $\nu-t$ respectively, and semilattices $S_{1}$ in $\mathcal{V}_{1}^{0}$ and $S_{2}$ in $\mathcal{V}_{2}^{0}$ so that

$$
\mathcal{V}^{0}=\mathcal{V}_{1}^{0} \oplus \mathcal{V}_{2}^{0}, \quad S=S_{1} \oplus \Lambda_{2} \quad \text { and } \quad L=k \Lambda_{1} \oplus S_{2},
$$

where $\Lambda_{1}:=\left\langle S_{1}\right\rangle$ and $\Lambda_{2}:=\left\langle S_{2}\right\rangle$. It is known that if $X_{\ell}=F_{4}$ or $G_{2}$, then $S_{1}$ and $S_{2}$ are lattices in $\mathcal{V}_{1}^{0}$ and $\mathcal{V}_{2}^{0}$, respectively. Also if $X_{\ell}=B_{\ell}$ (resp. $\left.X_{\ell}=C_{\ell}\right)$ with $\ell \geq 3$, then $S_{2}$ (resp. $\left.S_{1}\right)$ is a lattice in $\mathcal{V}_{2}^{0}$ (resp. $\mathcal{V}_{1}^{0}$ ). Therefore by [AABGP, Theorem 2.37 and Propositions 4.16 and 4.17],

$$
R=R\left(X_{\ell}, S_{1}, S_{2}\right):=(S+S) \cup\left(\dot{R}_{s h}+S_{1} \oplus \Lambda_{2}\right) \cup\left(\dot{R}_{l g}+k \Lambda_{1} \oplus S_{2}\right),
$$

with $S=S_{1}+\Lambda_{2}$.

Without loss of generality, we may write $\mathcal{B}:=\mathcal{B}_{1} \cup \mathcal{B}_{2}$ where

$$
\mathcal{B}_{1}:=\left\{\sigma_{1}, \ldots, \sigma_{t}\right\} \quad \text { and } \quad \mathcal{B}_{2}:=\left\{\sigma_{t+1}, \ldots, \sigma_{\nu}\right\}
$$


are bases of $S_{1}$ and $S_{2}$, respectively. For any subset $J$ of $\{1, \ldots, \nu\}$, we set

$$
\tau_{J}:=\sum_{r \in J} \sigma_{r}
$$

By (1.1) we have

$$
S_{j}=\bigcup_{J \in \operatorname{supp}_{\mathcal{B}_{j}}\left(S_{j}\right)}\left(\tau_{J}+2 \Lambda_{j}\right), \quad j=1,2 .
$$

Note that by the facts that $\mathcal{B}_{j} \subseteq S_{j}, 1 \leq j \leq 2$, and $\emptyset \in \operatorname{supp}_{\mathcal{B}_{j}}\left(S_{j}\right)$ we have

$\{\emptyset,\{r\} \mid 1 \leq r \leq t\} \subseteq \operatorname{supp}_{\mathcal{B}_{1}}\left(S_{1}\right)$ and $\{\emptyset,\{r\} \mid t+1 \leq r \leq \nu\} \subseteq \operatorname{supp}_{\mathcal{B}_{2}}\left(S_{2}\right)$.

We will see later how the terms $\operatorname{supp}_{\mathcal{B}_{j}}\left(S_{j}\right), 1 \leq j \leq 2$, appear in our presentation of the extended affine Weyl group of $R$ (see Theorem 3.1).

\section{$\S 2 . \quad$ A Finite Set of Generators for Extended Affine Weyl Group $\mathcal{W}$}

We keep all the notations as in Section 1. In particular, $R$ is an extended affine root system of reduced nonsimply laced type $X_{\ell}$ in $\mathcal{V}$ of nullity $\nu$ and of twist number $t$ satisfying (1.9). As in Section 1, we fix a complement $\dot{\mathcal{V}}$ of $\mathcal{V}^{0}$ in $\mathcal{V}$, a finite root system $\dot{R}$ in $\dot{\mathcal{V}}$, and two semilattices $S_{1}$ and $S_{2}$ in $\mathcal{V}^{0}$ such that (1.8), (1.9) and (1.12) hold. For a subset $J=\left\{i_{1}, \ldots, i_{n}\right\}$ of $\{1, \ldots, \nu\}$ with $i_{1}<i_{2}<\cdots<i_{n}$ and a group $G$ we make the convention

$$
\prod_{i \in J} a_{i}=a_{i_{1}} a_{i_{2}} \cdots a_{i_{n}} \quad\left(a_{i} \in G\right) .
$$

(Here we interpret the product on an empty index set to be 1.) Also for a group $G$, we denote the commutator $x^{-1} y^{-1} x y$ of two elements $x, y \in G$ by $[x, y]$, and denote the center of $G$ by $Z(G)$.

To introduce the extended affine Weyl group of $R$, we need to consider the so called a hyperbolic extension $\tilde{\mathcal{V}}$ of $\mathcal{V}$ as follows. Throughout our work we fix a basis $\left\{\lambda_{1}, \ldots, \lambda_{\nu}\right\}$ of the dual space $\left(\mathcal{V}^{0}\right)^{*}$ of $\mathcal{V}^{0}$ and we set $\tilde{\mathcal{V}}:=\mathcal{V} \oplus\left(\mathcal{V}^{0}\right)^{*}$. Now we extend the form $(\cdot, \cdot)$ on $\mathcal{V}$ to a non-degenerate form on $\tilde{\mathcal{V}}$, denoted again by $(\cdot, \cdot)$, as follows:

$$
\begin{aligned}
& -(., .))_{\mid \mathcal{V} \times \mathcal{v}}:=(., .), \\
& \text { - }\left(\dot{\mathcal{V}},\left(\mathcal{V}^{0}\right)^{*}\right)=\left(\left(\mathcal{V}^{0}\right)^{*},\left(\mathcal{V}^{0}\right)^{*}\right):=\{0\}, \\
& \text { - }\left(\sigma_{r}, \lambda_{s}\right):=\delta_{r, s}, \quad 1 \leq r, s \leq \nu .
\end{aligned}
$$


Let $\mathrm{O}(\tilde{\mathcal{V}})$ be the orthogonal group of $\tilde{\mathcal{V}}$, with respect to $I=(\cdot, \cdot)$. For each $\alpha \in \mathcal{V}^{\times}:=\{v \in \mathcal{V} \mid(v, v) \neq 0\}$, set $\alpha^{\vee}=2 \alpha /(\alpha, \alpha)$ and define $w_{\alpha} \in \mathrm{O}(\tilde{\mathcal{V}})$ by

$$
w_{\alpha}(u)=u-\left(u, \alpha^{\vee}\right) \alpha, \quad(u \in \tilde{\mathcal{V}}) .
$$

One can see easily for $\alpha \in \mathcal{V}^{\times}$and $w \in \mathrm{O}(\tilde{\mathcal{V}})$ that

$$
w_{\alpha}^{2}=1 \quad \text { and } \quad w w_{\alpha} w^{-1}=w_{w(\alpha)} .
$$

Definition 2.1. $\quad$ The extended affine Weyl group $\mathcal{W}$ of $R$ is the subgroup of the orthogonal group $\mathrm{O}(\tilde{\mathcal{V}})$ generated by the reflections $w_{\alpha}, \alpha \in R^{\times}$. Using the fact that $\dot{R} \subseteq R$, we identify the finite Weyl group $\dot{\mathcal{W}}$ of $\dot{R}$ with a subgroup of $\mathcal{W}$. The subgroup

$$
\mathcal{H}:=\left\langle w_{\alpha+\sigma} w_{\alpha} \mid \alpha \in R^{\times}, \sigma \in \mathcal{V}^{0}, \alpha+\sigma \in R\right\rangle
$$

of $\mathcal{W}$ is called the Heisenberg-like group of $R$.

We know from [Az1, Proposition 3.27] that $\mathcal{W}$ is the semidirect product of a finite Weyl group and $\mathcal{H}$ (we see this fact later as a byproduct of our results). Since finite Weyl groups are completely known, for a better understanding of $\mathcal{W}$, one should study $\mathcal{H}$. This is what we start with. Let us first introduce a few notation.

Throughout this work we fix a fundamental basis

$$
\dot{\Pi}=\left\{\alpha_{1}, \ldots, \alpha_{\ell}\right\}
$$

of $\dot{R}$. Moreover, we normalize the form $(\cdot, \cdot)$ such that

$$
(\alpha, \alpha)=2 \quad \text { if } \alpha \in \dot{R}_{s h} \quad \text { and } \quad(\alpha, \alpha)=2 k \quad \text { if } \alpha \in \dot{R}_{l g} .
$$

For any $\alpha \in R^{\times}, 1 \leq i \leq \ell$ and $1 \leq r \leq \nu$, set

$$
k(\alpha):=\frac{2 k}{(\alpha, \alpha)}=\left\{\begin{array}{l}
k, \text { if } \alpha \in \dot{R}_{s h}+S, \\
1, \text { if } \alpha \in \dot{R}_{l g}+L,
\end{array} \quad k_{r}:=\left\{\begin{array}{l}
k, \text { if } 1 \leq r \leq t, \\
1, \text { if } t<r \leq \nu
\end{array}\right.\right.
$$

and

$$
k_{i, r}=k\left(\alpha_{i}, \sigma_{r}\right):=\min \left\{n \in \mathbb{N} \mid \alpha_{i}+n \sigma_{r} \in R\right\} .
$$

Then from (1.9), it follows that

$$
\alpha_{i}+\mathbb{Z} k_{i, r} \sigma_{r} \subseteq R, \quad \text { and } \quad k_{i, r}= \begin{cases}1, & \text { if } \alpha_{i} \in \dot{R}_{s h}, \\ k_{r}, & \text { if } \alpha_{i} \in \dot{R}_{l g}\end{cases}
$$


For $\alpha \in \mathcal{V}$ and $\sigma \in \mathcal{V}^{0}$ we define a linear map $T_{\alpha}^{\sigma} \in \operatorname{End}(\tilde{\mathcal{V}})$ by

$$
T_{\alpha}^{\sigma}(u):=u+(\alpha, u) \sigma-(\sigma, u) \alpha-\frac{(\alpha, \alpha)}{2}(\sigma, u) \sigma \quad(u \in \tilde{\mathcal{V}}) .
$$

The following lemma will be frequently referred to in the sequel.

Lemma 2.1 ([AzS2, Lemma 1.1]). Let $w \in O(\mathcal{V}), \alpha, \beta, \gamma \in \mathcal{V}$ and $\sigma, \delta$, $\tau \in \mathcal{V}^{0}$. Then
(i) $T_{\alpha}^{r \sigma}=T_{r \alpha}^{\sigma}, r \in \mathbb{R}$,
(ii) $T_{\alpha}^{\sigma+\delta}=T_{\alpha}^{\sigma} T_{\alpha}^{\delta} T_{\delta}^{(\alpha, \alpha) \frac{\sigma}{2}}$,
(iii) $T_{\alpha+\beta}^{\sigma}=T_{\alpha}^{\sigma} T_{\beta}^{\sigma}$,
(iv) $\left[T_{\alpha}^{\sigma}, T_{\beta}^{\delta}\right]=T_{\sigma}^{(\alpha, \beta) \delta}$,
(v) $\left[T_{\alpha}^{\sigma} T_{\beta}^{\delta}, T_{\gamma}^{\tau}\right]=\left[T_{\alpha}^{\sigma}, T_{\gamma}^{\tau}\right]\left[T_{\beta}^{\delta}, T_{\gamma}^{\mu}\right]$,
(vi) $w T_{\alpha}^{\sigma} w^{-1}=T_{w(\alpha)}^{\sigma}$,
(vii) $T_{\alpha^{\vee}}^{\sigma}=w_{\alpha+\sigma} w_{\alpha}, \alpha \in \mathcal{V}^{\times}$,
(viii) $\left(T_{\sigma}^{\delta}\right)^{-1}=T_{\delta}^{\sigma}$.

For any $1 \leq i \leq \ell$ and $1 \leq r, s \leq \nu$ we set

$$
c_{r, s}:=T_{\sigma_{r}}^{k^{-1} \sigma_{s}} \quad \text { and } \quad t_{i, r}:=T_{\alpha_{i}^{\vee}}^{k_{i, r} \sigma_{r}} .
$$

It is clear that $c_{r, s}=c_{s, r}^{-1}$ and $c_{r, r}=1$ for all $1 \leq r, s \leq \nu$. Also from (2.8), (2.6) and parts (iv) and (vi) of Lemma 2.1, it follows that

$$
c_{r, s} w=w c_{r, s}, \quad(w \in \mathcal{W}), \quad t_{i, r}=w_{\alpha_{i}+k_{i, r} \sigma_{r}} w_{\alpha_{i}} \in \mathcal{H}
$$

and

$$
\left[t_{i, r}, t_{j, s}\right]=c_{r, s}^{\left(\alpha_{i}, \alpha_{j}^{\vee}\right) k\left(\alpha_{i}\right) k_{i, r} k_{j, s}} \quad(1 \leq j \leq \ell) .
$$

Finally, using (2.7) and (2.8) we have

$$
\prod_{r \in J} t_{i, r}^{n_{r}}\left(\alpha_{j}\right)=\alpha_{j}+\sum_{r \in J}\left(\alpha_{j}, \alpha_{i}^{\vee}\right) n_{r} k_{i, r} \sigma_{r} \quad\left(1 \leq i, j \leq \ell, n_{r} \in \mathbb{Z}\right) .
$$

Let $\delta_{j}, j=1,2$ be the supporting function of the semilattice $S_{j}$, relative to the basis $\mathcal{B}_{j}$ (see (1.2) and (1.10)). For any $1 \leq r, s \leq \nu$ we set

$$
\Delta(r, s)= \begin{cases}\delta_{1}(r, s) & \text { if } 1 \leq r, s \leq t, \\ 1 & \text { if } 1 \leq r \leq t<s \leq \nu \\ -1 & \text { if } 1 \leq s \leq t<r \leq \nu \\ \delta_{2}(r, s) & \text { if } t<r, s \leq \nu .\end{cases}
$$


It is clear that $\Delta(r, s)=-\Delta(s, r)$ for all $1 \leq r<s \leq \nu$. If $S_{1}$ (resp. $\left.S_{2}\right)$ is a lattice, in particular, if $X_{\ell}=C_{\ell}(\ell \geq 3), F_{4}$ or $G_{2}$ (resp. $X_{\ell}=B_{\ell}(\ell \geq 3), F_{4}$ or $G_{2}$ ), then $\Delta(r, s)=1$ for all $1 \leq r<s \leq t$ (resp. $t<r<s \leq \nu$ ).

In order to describe the center $Z(\mathcal{H})$, for any $J \subseteq\{1, \ldots, \nu\}$, we set

$$
z_{J}= \begin{cases}c_{r, s}^{\Delta(r, s) k_{r}}, & \text { if } J=\{r, s\}, r<s, \\ \prod_{\{r, s \in J \mid r<s\}} c_{r, s}^{k_{r}}, & \text { if } J \in \operatorname{supp}_{\mathcal{B}_{1}}\left(S_{1}\right) \cup \operatorname{supp}_{\mathcal{B}_{2}}\left(S_{2}\right), \\ 1, & \text { otherwise. }\end{cases}
$$

Then from (2.9) we have

$$
w z_{J}=z_{J} w \quad \text { for any } w \in \mathcal{W} .
$$

Lemma 2.2. (i) $C:=\left\langle c_{r, s} \mid 1 \leq r<s \leq \nu\right\rangle$ and $\left\langle z_{\{r, s\}} \mid 1 \leq r<s \leq \nu\right\rangle$ are free abelian subgroups of $O(\tilde{\mathcal{V}})$ of rank $\nu(\nu-1) / 2$ on generators $c_{r, s}$ and $z_{\{r, s\}}$, respectively, $1 \leq r<s \leq \nu$.

(ii) $\left\langle z_{J} \mid J \subseteq\{1, \ldots, \nu\}\right\rangle$ is a free abelian subgroup of $O(\tilde{\mathcal{V}})$ of rank $\nu(\nu-1) / 2$.

Proof. (i) holds by (2.8), (2.13) and [AzS2, Lemma 1.7(ii)]. Next, suppose that $C^{\prime}:=\left\langle c_{r, s}^{2 k}: 1 \leq r<s \leq \nu\right\rangle$. Then from (2.13), we see that the group in the statement of (ii) is squeezed between two groups $C^{\prime}$ and $C$ and so by (i), the result follows.

Lemma 2.3. (i) If $(\alpha, J) \in\left(\dot{R}_{s h} \times \operatorname{supp}_{\mathcal{B}_{1}}\left(S_{1}\right)\right) \cup\left(\dot{R}_{l g} \times \operatorname{supp}_{\mathcal{B}_{2}}\left(S_{2}\right)\right)$, then

$$
z_{J}=w_{\alpha} w_{\alpha+\tau_{J}} \prod_{r \in J} w_{\alpha+\sigma_{r}} w_{\alpha} \in Z(\mathcal{W}) \cap \mathcal{H}
$$

(ii) If $(\alpha, \beta) \in \dot{R}_{s h} \times \dot{R}_{l g}$ so that $(\alpha, \beta) \neq 0$ and $\{r, s\} \notin \operatorname{supp}_{B_{1}}\left(S_{1}\right) \cup$ supp $_{B_{2}}\left(S_{2}\right)$ with $1 \leq r<s \leq \nu$, then $z_{\{r, s\}} \in Z(\mathcal{W}) \cap \mathcal{H}$ and

$$
z_{\{r, s\}}= \begin{cases}{\left[w_{\alpha+\sigma_{r}} w_{\alpha}, w_{\alpha+\sigma_{s}} w_{\alpha}\right],} & \text { if } 1 \leq r<s \leq t, \\ {\left[w_{\beta+\sigma_{r}} w_{\beta}, w_{\beta+\sigma_{s}} w_{\beta}\right],} & \text { if } t<r<s \leq \nu \\ {\left[w_{\beta+\sigma_{s}} w_{\beta}, w_{\alpha+\sigma_{r}} w_{\alpha}\right],} & \text { if } 1 \leq r \leq t<s \leq \nu .\end{cases}
$$

Proof. (i) From (1.9) and (1.12), it follows that $\alpha+\tau_{J} \in R^{\times}$and $\alpha+\sigma_{r} \in$ $R^{\times}$, for all $r \in J$ and so $w_{\alpha} w_{\alpha+\tau_{J}} \prod_{r \in J} w_{\alpha+\sigma_{r}} w_{\alpha} \in \mathcal{H}$. Then from the facts 
that $k_{r}=k(\alpha)$ for any $r \in J$ and $k^{-1} k(\alpha)=\left(\alpha^{\vee}, \alpha^{\vee}\right) / 2$, we have

$$
\begin{aligned}
& z_{J}=\left(\prod_{r \in J} w_{\alpha+\sigma_{r}} w_{\alpha}\right)^{-1} z_{J} \prod_{r \in J} w_{\alpha+\sigma_{r}} w_{\alpha} \quad(\text { by }(2.14)) \\
& =\left(\prod_{r \in J} w_{\alpha+\sigma_{r}} w_{\alpha}\right)^{-1} \prod_{\{r, s \in J \mid r<s\}} c_{r, s}^{k_{r}} \prod_{r \in J} w_{\alpha+\sigma_{r}} w_{\alpha} \quad(\operatorname{by}(2.13)) \\
& =\left(\prod_{r \in J} w_{\alpha+\sigma_{r}} w_{\alpha}\right)^{-1} \prod_{\{r, s \in J \mid r<s\}} c_{r, s}^{k(\alpha)} \prod_{r \in J} w_{\alpha+\sigma_{r}} w_{\alpha} \\
& =\left(\prod_{r \in J} w_{\alpha+\sigma_{r}} w_{\alpha}\right)^{-1} \prod_{\{r, s \in J \mid r<s\}} T_{\sigma_{r}}^{k^{-1} k(\alpha) \sigma_{s}} \prod_{r \in J} w_{\alpha+\sigma_{r}} w_{\alpha} \quad \text { (by (2.8)) } \\
& =\left(\prod_{r \in J} w_{\alpha+\sigma_{r}} w_{\alpha}\right)^{-1}\left(\prod_{\{r, s \in J \mid r<s\}} T_{\sigma_{s}}^{k^{-1} k(\alpha) \sigma_{r}}\right)^{-1} \prod_{r \in J} w_{\alpha+\sigma_{r}} w_{\alpha} \\
& \text { (by Lemma 2.1(viii)) } \\
& =\left(\prod_{r \in J} T_{\alpha^{v}}^{\sigma_{r}}\right)^{-1}\left(\prod_{\{r, s \in J \mid r<s\}} T_{\sigma_{s}}^{k^{-1} k(\alpha) \sigma_{r}}\right)^{-1} \prod_{r \in J} w_{\alpha+\sigma_{r}} w_{\alpha} \quad \text { (by Lemma 2.1(vii)) } \\
& =\left(\prod_{r \in J} T_{\alpha^{\vee}}^{\sigma_{r}} \prod_{\{r, s \in J \mid r<s\}} T_{\sigma_{s}}^{k^{-1} k(\alpha) \sigma_{r}}\right)^{-1} \prod_{r \in J} w_{\alpha+\sigma_{r}} w_{\alpha} \quad \text { (by Lemma 2.1(iv)) } \\
& =\left(T_{\alpha^{\vee}}^{\tau_{J}}\right)^{-1} \prod_{r \in J} w_{\alpha+\sigma_{r}} w_{\alpha} \quad \text { (by [AzS2, Lemma 1.2]) } \\
& =\left(w_{\alpha+\tau_{J}} w_{\alpha}\right)^{-1} \prod_{r \in J} w_{\alpha+\sigma_{r}} w_{\alpha} \quad \text { (by Lemma } 2.1(\text { vii) }) \\
& =w_{\alpha} w_{\alpha+\tau_{J}} \prod_{r \in J} w_{\alpha+\sigma_{r}} w_{\alpha} .
\end{aligned}
$$

Thus by (2.14) we have $z_{J} \in Z(\mathcal{W}) \cap \mathcal{H}$.

(ii) By the way $\Delta(r, s)$ and $k_{r}$ are defined and the facts that $(\alpha, \alpha)=2$, $(\beta, \beta)=2 k$ and $(\alpha, \beta)=-k$ we have

$$
\begin{aligned}
z_{\{r, s\}}= & c_{r, s}^{\Delta(r, s) k_{r}} \quad(\text { using }(2.13)) \\
= & \begin{cases}c_{r, s}^{2 k}, \text { if } \quad 1 \leq r<s \leq t, \\
c_{r, s}^{2}, \text { if } \quad t+1 \leq r<s \leq \nu, \\
c_{r, s}^{k}, \text { if } 1 \leq r \leq t<s \leq \nu,\end{cases} \\
= & \begin{cases}T_{\sigma_{r}}^{2 \sigma_{s}}, \quad \text { if } 1 \leq r<s \leq t, \\
\left.T_{\sigma_{r}}^{2 k^{-1} \sigma_{s}}, \text { if } t+1 \leq r<s \leq \nu, \quad \text { (using }(2.8)\right) \\
T_{\sigma_{r}}^{\sigma_{s}}, \quad \text { if } 1 \leq r \leq t<s \leq \nu,\end{cases}
\end{aligned}
$$




$$
\begin{aligned}
& =\left\{\begin{array}{l}
{\left[T_{\alpha^{v}}^{\sigma_{r}}, T_{\alpha^{v}}^{\sigma_{s}}\right], \text { if } \quad 1 \leq r<s \leq t,} \\
{\left[T_{\beta^{v}}^{\sigma_{r}}, T_{\beta^{v}}^{\sigma_{s}}\right], \text { if } t+1 \leq r<s \leq \nu, \quad \text { (using Lemma 2.1(iv)) }} \\
{\left[T_{\beta^{v}}^{\sigma_{s}}, T_{\alpha^{v}}^{\sigma_{r}}\right], \text { if } \quad 1 \leq r \leq t<s \leq \nu,}
\end{array}\right. \\
& =\left\{\begin{array}{l}
{\left[w_{\alpha+\sigma_{r}} w_{\alpha}, w_{\alpha+\sigma_{s}} w_{\alpha}\right], \text { if } 1 \leq r<s \leq t,} \\
{\left[w_{\beta+\sigma_{r}} w_{\beta}, w_{\beta+\sigma_{s}} w_{\beta}\right], \text { if } t+1 \leq r<s \leq \nu, \quad \text { (using Lemma 2.1(vii)). }} \\
{\left[w_{\beta+\sigma_{s}} w_{\beta}, w_{\alpha+\sigma_{r}} w_{\alpha}\right], \text { if } 1 \leq r \leq t<s \leq \nu,}
\end{array}\right.
\end{aligned}
$$

Therefore $z_{\{r, s\}} \in Z(\mathcal{W}) \cap \mathcal{H}($ see $(2.14))$.

Let $a_{i j}=\left(\alpha_{i}, \alpha_{j}^{\vee}\right)$, the $(i, j)$-entry of the Cartan matrix of $\dot{R}$ with respect to $\dot{\Pi}$ (see (2.3)). Then for $1 \leq i, j \leq \ell$ and $1 \leq r \leq s \leq \nu$, we put

$$
a_{i, j}(r):=k_{j, r} k_{i, r}^{-1} a_{i, j} \quad \text { and } \quad a_{i, j}(r, s):=k_{r}^{-1} k\left(\alpha_{i}\right) k_{i, r} k_{j, s} a_{i, j} .
$$

Note that $a_{i, j} \in\{-1,0,2,-k\}$. Moreover, if $\left|a_{i, j}\right|=\left|a_{j, i}\right| \neq 0$, then $\left|\alpha_{i}\right|=\left|\alpha_{j}\right|$ and if $\left|a_{i, j}\right|<\left|a_{j, i}\right|$ then $a_{i, j}=-1, a_{j, i}=-k,\left|\alpha_{i}\right|<\left|\alpha_{j}\right|$ and $k\left(\alpha_{i}\right)=k$. So from (2.6) and (2.15) we have (for $1 \leq r \leq s \leq \nu$ )

$$
a_{i, j}(r)= \begin{cases}-k_{r}, & \text { if }\left|a_{i, j}\right|<\left|a_{j, i}\right|, \\ a_{i, j}, & \text { if }\left|a_{i, j}\right|=\left|a_{j, i}\right|, \\ -k k_{r}^{-1}, & \text { if }\left|a_{i, j}\right|>\left|a_{j, i}\right|,\end{cases}
$$

and

$$
a_{i, j}(r, s)= \begin{cases}-k k_{r}^{-1} k_{s}, & \text { if }\left|a_{i, j}\right|<\left|a_{j, i}\right|, \\ k k_{r}^{-1} a_{i, j}, & \text { if }\left|a_{i, j}\right|=\left|a_{j, i}\right|, \alpha_{i} \in \dot{R}_{s h}, \\ k_{s} a_{i, j}, & \text { if }\left|a_{i, j}\right|=\left|a_{j, i}\right|, \alpha_{i} \in \dot{R}_{l g}, \\ -k, & \text { if }\left|a_{i, j}\right|>\left|a_{j, i}\right|,\end{cases}
$$

where $k$ and $k_{r}, 1 \leq r \leq \nu$ are defined by (1.7) and (2.5), respectively. Then from $(2.16),(2.17)$ and the fact that $k k_{r}^{-1} \in\{1, k\}$ for any $1 \leq r \leq \nu$ we have

$$
a_{i, j}(r) \in \mathbb{Z} \quad \text { and } \quad a_{i, j}(r, s) \in \mathbb{Z} .
$$

Lemma 2.4. If $1 \leq i, j \leq \ell$ and $1 \leq r \leq s \leq \nu$, then

$$
\Delta(r, s)^{-1} a_{i, j}(r, s) \in \mathbb{Z} .
$$


Proof. First, let $X_{\ell}=F_{4}$ or $G_{2}$. Then from (2.18) and the fact that $S_{1}$ and $S_{2}$ are lattices we have $\Delta(r, s)^{-1} a_{i, j}(r, s)=a_{i, j}(r, s) \in \mathbb{Z}$. Next, let $X_{\ell}=B_{\ell}$ or $C_{\ell}$. From (1.2) we know that $\Delta(r, s)=1$ if $1 \leq r \leq t<s \leq \nu$. Also if $X=B_{\ell}(\ell \geq 3)$ and $t<r \leq s \leq \nu$ then $S_{2}$ is a lattice and if $X=C_{\ell}$ $(\ell \geq 3)$ and $1 \leq r \leq s \leq t$, then $S_{1}$ is a lattice, so $\Delta(r, s)=1$. Therefore the only cases which we must consider are

(1) $X=B_{\ell}, 1 \leq r<s \leq t$,

(2) $X=C_{\ell}, t<r<s \leq \nu,\left(B_{2} \equiv C_{2}\right)$.

Since $\Delta(r, s)^{-1} \in\{1,1 / 2\}$, it is enough to show that if $\Delta^{-1}(r, s)=1 / 2$ then $a_{i, j}(r, s) \in 2 \mathbb{Z}$. In case (1) we have $k_{r}=k_{s}=k=2$ and so by $(2.17), a_{i, j}(r, s)$ is even except possibly when $\left|a_{i, j}\right|=\left|a_{j, i}\right| \neq 0$ and $\alpha_{i}, \alpha_{j} \in \dot{R}_{s h}$. But in type $B_{\ell}$ this only can happen if $\alpha_{i}=\alpha_{j}$. Then $a_{i, j}=2$ and $a_{i, j}(r, s) \in 2 \mathbb{Z}$. In case (2), we have $k_{r}=k_{s}=1$. Then by $(2.17), a_{i, j}(r, s) \in 2 \mathbb{Z}$ except possibly when $\left|a_{i, j}\right|=\left|a_{j, i}\right| \neq 0$ and $\alpha_{i}=\alpha_{j} \in \dot{R}_{l g}$. Again this only can happen if $\alpha_{i}=\alpha_{j}$. Then $a_{i, j}=2$ and we are done.

Lemma 2.5. (i) If $1 \leq i, j \leq \ell$ and $1 \leq r \leq s \leq \nu$, then

$$
\left[t_{i, r}, t_{j, s}\right]=z_{\{r, s\}}^{\Delta(r, s)^{-1} a_{i, j}(r, s)} \quad \text { and } \quad w_{\alpha_{i}} t_{j, r} w_{\alpha_{i}}=t_{j, r} t_{i, r}^{-a_{i, j}(r)} .
$$

(ii) If $J \in \operatorname{supp}_{\mathcal{B}_{1}}\left(S_{1}\right) \cup \operatorname{supp}_{\mathcal{B}_{2}}\left(S_{2}\right)$, then

$$
z_{J}^{2}=\prod_{\{r, s \in J: r<s\}} z_{\{r, s\}}^{3-\Delta(r, s)} .
$$

(iii) If $J \in \operatorname{supp}_{\mathcal{B}_{j}}\left(S_{j}\right) \backslash \mathcal{J}_{\mathcal{B}_{j}}\left(S_{j}\right), j=1,2$, then

$$
z_{J}=\prod_{\{r, s \in J} z_{\{r, s\}} .
$$

Proof. (i) We have

$$
\begin{aligned}
{\left[t_{i, r}, t_{j, s}\right] } & =c_{r, s}^{\left(\alpha_{i}, \alpha_{j}^{\vee} k\left(\alpha_{i}\right) k_{i, r} k_{j, s}\right.} \quad(\text { by }(2.10)) \\
& =c_{r, s}^{\Delta(r, s) k_{r} \Delta(r, s)^{-1} a_{i, j}(r, s)} \quad(\text { by }(2.15)) \\
& =\left(c_{r, s}^{\Delta(r, s) k_{r}}\right)^{\Delta(r, s)^{-1} a_{i, j}(r, s)} \quad(\text { by }(2.19)) \\
& =z_{\{r, s\}}^{\Delta(r, s)^{-1} a_{i, j}(r, s)} \quad(\text { by }(2.13))
\end{aligned}
$$

and

$$
w_{\alpha_{i}} t_{j, r} w_{\alpha_{i}}=w_{\alpha_{i}} T_{\alpha_{j}^{\vee}}^{k_{j, r} \sigma_{r}} w_{\alpha_{i}} \quad(\text { by }(2.8))
$$




$$
\begin{aligned}
& =T_{w_{\alpha_{i}}\left(\alpha_{j}^{\vee}\right)}^{k_{j, r} \sigma_{r}}=T_{\alpha_{j}^{\vee}-\left(\alpha_{j}^{\vee}, \alpha_{i}^{\vee}\right) \alpha_{i}}^{k_{j, r} \sigma_{r}} \quad \text { (by Lemma 2.1(vi)) } \\
& =T_{\alpha_{j}^{\vee}}^{k_{j, r} \sigma_{r}} T_{-\left(\alpha_{j}^{\vee}, \alpha_{i}\right) \alpha_{i}^{\vee}}^{k_{j, r} \sigma_{r}} \quad(\text { by Lemma 2.1(iii)) } \\
& =t_{j, r} T_{-\left(\alpha_{j}^{\vee}, \alpha_{i}\right) \alpha_{i}^{\vee}}^{k_{j, r} \sigma^{\vee}} \quad(\text { by }(2.8)) \\
& =t_{j, r} T_{\alpha_{i}^{\vee}}^{-k_{j, r}\left(\alpha_{j}^{\vee}, \alpha_{i}\right) \sigma_{r}} \quad(\text { by Lemma 2.1(i)) } \\
& =t_{j, r} T_{\alpha_{i}^{\vee}}^{-k_{j, r} k_{i, r}^{-1}\left(\alpha_{j}^{\vee}, \alpha_{i}\right) k_{i, r} \sigma_{r}} \\
& =t_{j, r} T_{\alpha_{i}^{\vee}}^{-a_{i, j}(r) k_{i, r} \sigma_{r}} \quad(\text { by }(2.15)) \\
& =t_{j, r}\left(T_{\alpha_{i}^{v}}^{k_{i, r} \sigma_{r}}\right)^{-a_{i, j}(r)} \quad(\text { by }(2.18)) \\
& =t_{j, r} t_{i, r}^{-a_{i, j}(r)} .
\end{aligned}
$$

(ii) From (2.13) and the fact that $(3-\Delta(r, s)) \Delta(r, s)=2$ for $r<s$, we obtain

$$
\prod_{\{r, s \in J: r<s\}} z_{\{r, s\}}^{3-\Delta(r, s)}=\prod_{\{r, s \in J: r<s\}} c_{r, s}^{(3-\Delta(r, s)) \Delta(r, s) k_{r}}=\prod_{\{r, s \in J: r<s\}} c_{r, s}^{2 k_{r}}=z_{J}^{2} .
$$

(iii) Since $\{r, s\} \in \operatorname{supp}_{\mathcal{B}_{1}}\left(S_{1}\right) \cup \operatorname{supp}_{\mathcal{B}_{2}}\left(S_{2}\right)$, for all $r, s \in J$, then $\Delta(r, s)=$ 1 for all $r, s \in J$, and so from (2.13) we get

$$
\prod_{\{r, s \in J: r<s\}} z_{\{r, s\}}=\prod_{\{r, s \in J: r<s\}} c_{r, s}^{\Delta(r, s) k_{r}}=\prod_{\{r, s \in J: r<s\}} c_{r, s}^{k_{r}}=z_{J} .
$$

To obtain further information about elements of $Z(\mathcal{H})$, we consider pairs of collections of the form

$$
(\underline{m}, \underline{\epsilon})=\left(\left\{m_{r, s}\right\}_{1 \leq r<s \leq t},\left\{\epsilon_{J}\right\}_{J \in \mathcal{J}_{\mathcal{B}_{1}}\left(S_{1}\right)}\right)
$$

and

$$
(\bar{n}, \bar{\varepsilon})=\left(\left\{n_{r, s}\right\}_{t<r<s \leq \nu},\left\{\varepsilon_{J}\right\}_{J \in \mathcal{J}_{\mathcal{B}_{2}}\left(S_{2}\right)}\right),
$$

where $m_{r, s}, n_{r, s} \in \mathbb{Z}$ and $\epsilon_{J} \in\{0,1\}$. To each such pair we assign central elements of $\mathcal{H}$ by

$$
\underline{u}(\underline{m}, \underline{\epsilon})=\prod_{1 \leq r<s \leq t} z_{\{r, s\}}^{m_{r, s}} \prod_{J \in \mathcal{J}_{\mathcal{B}_{1}}\left(S_{1}\right)} z_{J}^{\epsilon_{J}}
$$

and

$$
\bar{u}(\bar{n}, \bar{\varepsilon})=\prod_{t<r<s \leq \nu} z_{\{r, s\}}^{n_{r, s}} \prod_{J \in \mathcal{J}_{\mathcal{B}_{2}}\left(S_{2}\right)} z_{J}^{\varepsilon_{J}} .
$$


Lemma 2.6. (i) Each element $u \in\left\langle z_{J} \mid J \subseteq\{1, \ldots, t\}\right\rangle$ has an expression of the form $u=\underline{u}(\underline{m}, \underline{\epsilon})$, where pair $(\underline{m}, \underline{\epsilon})$ is of the form (2.20). Moreover, $\underline{u}(\underline{m}, \underline{\epsilon})=1$ if and only if $(\underline{m}, \underline{\epsilon})$ satisfies

$$
\begin{gathered}
m_{r, s}=-\Delta(r, s)^{-1} \sum_{J \in \mathcal{J}_{\mathcal{B}_{1}}\left(S_{1}\right)} \chi_{J}(r, s) \epsilon_{J}, \quad 1 \leq r<s \leq t, \quad \text { where } \\
\chi_{J}(r, s)=\left\{\begin{array}{l}
1, \text { if }\{r, s\} \subsetneq J, \\
0, \text { otherwise. }
\end{array}\right.
\end{gathered}
$$

(ii) Each element $u \in\left\langle z_{J} \mid J \subseteq\{t+1, \ldots, \nu\}\right\rangle$ has an expression of the form $u=\bar{u}(\bar{n}, \bar{\varepsilon})$, where pair $(\bar{n}, \bar{\varepsilon})$ is of the form (2.21). Moreover, $\bar{u}(\bar{n}, \bar{\varepsilon})=1$ if and only if $(\bar{n}, \bar{\varepsilon})$ satisfies

$$
n_{r, s}=-\Delta(r, s)^{-1} \sum_{J \in \mathcal{J}_{\mathcal{B}_{2}}\left(S_{2}\right)} \chi_{J}(r, s) \varepsilon_{J}, \quad t<r<s \leq \nu .
$$

Proof. (i) Let $u \in\left\langle z_{J} \mid J \subseteq\{1, \ldots, t\}\right\rangle$. Using Lemma 2.5(ii) it is immediate that $u$ can be written in the form $\underline{u}(\underline{m}, \underline{\epsilon})$. Moreover from the way $z_{J}$ is defined we obtain

$$
\begin{aligned}
\underline{u}(\underline{m}, \underline{\epsilon}) & =\prod_{1 \leq r<s \leq t} z_{\{r, s\}}^{m_{r, s}} \prod_{J \in \mathcal{J}_{\mathcal{B}_{1}}\left(S_{1}\right)} z_{J}^{\epsilon_{J}} \\
& =\prod_{1 \leq r<s \leq t} c_{r, s}^{k \Delta(r, s) m_{r, s}} \prod_{J \in \mathcal{J}_{\mathcal{B}_{1}}\left(S_{1}\right)} \prod_{\{r, s \in J \mid r<s\}} c_{r, s}^{k \epsilon_{J}} \\
& =\prod_{1 \leq r<s \leq t} c_{r, s}^{k \Delta(r, s) m_{r, s}} \prod_{1 \leq r<s \leq t} \prod_{J \in \mathcal{J}_{\mathcal{B}_{1}}\left(S_{1}\right)} c_{r, s}^{k \chi_{J}(r, s) \epsilon_{J}} \\
& =\prod_{1 \leq r<s \leq t} c_{r, s}^{k \Delta(r, s) m_{r, s}} \prod_{\substack{k_{J_{J}} \\
\mathcal{J}_{\mathcal{B}_{1}}\left(S_{1}\right)}} \chi_{J}(r, s) \epsilon_{J} \\
& =\prod_{1 \leq r<s \leq s \leq t} c_{r, s}^{k \Delta(r, s) m_{r, s}+k \sum_{J \in \mathcal{J}_{\mathcal{B}}\left(S_{1}\right)} \chi_{J}(r, s) \epsilon_{J}} .
\end{aligned}
$$

Then from Lemma 2.2(i) and the fact that $m_{r, s} \in \mathbb{Z}$ for all $1 \leq r \leq s \leq t$, it follows that $\underline{u}(\underline{m}, \underline{\epsilon})=1$ if and only if $(\underline{m}, \underline{\epsilon})$ satisfies $(2.24)$.

(ii) An argument analogous to (i) shows that (ii) holds.

The results of this section are summarized in the following propositions. For completeness we have recorded parts (iii)-(iv) and (vii)-(viii) which can be found in $[\mathrm{Az} 1]$ and $[\mathrm{K}]$. The proofs of these parts are now easy consequences of our results in this section. 


\section{Proposition 2.1. (i)}

$$
\mathcal{H}=\left\langle t_{i, r}, z_{J} \mid 1 \leq i \leq \ell, 1 \leq r \leq \nu, J \in \mathcal{J}_{\mathcal{B}_{1}}\left(S_{1}\right) \cup \mathcal{J}_{\mathcal{B}_{2}}\left(S_{2}\right)\right\rangle .
$$

(ii) $\mathcal{W}=\left\langle w_{\alpha_{i}}, t_{i, r}, z_{J} \mid 1 \leq i \leq \ell, 1 \leq r \leq \nu, J \in \mathcal{J}_{\mathcal{B}_{1}}\left(S_{1}\right) \cup \mathcal{J}_{\mathcal{B}_{2}}\left(S_{2}\right)\right\rangle$.

(iii) $\mathcal{H}$ is a torsion free, two-step nilpotent group.

(iv) $\mathcal{W}=\dot{\mathcal{W}} \ltimes \mathcal{H}$.

(v) Each $w \in \mathcal{W}$ has a unique expression of the form

$$
w=w\left(\dot{w},\left\{n_{i, r}\right\}, u_{1},\left\{m_{r, s}\right\}, u_{2}\right):=\dot{w} \prod_{r=1}^{\nu} \prod_{i=1}^{\ell} t_{i, r}^{n_{i, r}} u_{1} \prod_{1 \leq r \leq t<s \leq \nu} z_{\{r, s\}}^{m_{r, s}} u_{2},
$$

where $u_{1} \in\left\langle z_{J} \mid J \subseteq\{1, \ldots, t\}\right\rangle, u_{2} \in\left\langle z_{J} \mid J \subseteq\{t+1, \ldots, \nu\}\right\rangle, m_{r, s}, n_{i, r} \in \mathbb{Z}$ and $\dot{w} \in \dot{\mathcal{W}}$. Moreover, $u_{1}$ and $u_{2}$ are of the forms $u_{1}=\underline{u}(\underline{m}, \underline{\epsilon})$ and $u_{2}=$ $\bar{u}(\bar{n}, \bar{\varepsilon})$, where $(\underline{m}, \underline{\epsilon})$ and $(\bar{n}, \bar{\varepsilon})$ are pairs of the forms (2.20) and (2.21), respectively.

(vi) $Z(\mathcal{W})=\left\langle z_{\{r, s\}}, z_{J} \mid 1 \leq r<s \leq \nu, J \in \mathcal{J}_{\mathcal{B}_{1}}\left(S_{1}\right) \cup \mathcal{J}_{\mathcal{B}_{2}}\left(S_{2}\right)\right\rangle$.

(vii) If $\nu=1$, then $Z(\mathcal{W})=\{1\}$ and $\mathcal{H}$ is a free abelian group of rank $\ell$.

(viii) If $\nu \geq 2$, then $Z(\mathcal{W})=Z(\mathcal{H})$ is a free abelian group of rank $\nu(\nu-1) / 2$.

Proof. (i)-(ii) Let $T$ and $T^{\prime}$ be the groups on the right hand sides of the statements (i) and (ii), respectively. From (2.9) and Lemma 2.3 we have $T \subseteq \mathcal{H}$ and $T^{\prime} \subseteq \mathcal{W}$. So to complete the proofs of (i) and (ii), it is enough to show that $w_{\alpha+\sigma} w_{\alpha} \in T$ and $w_{\alpha} \in T^{\prime}$ for all $\alpha \in R^{\times}$and $\sigma \in \mathcal{V}^{0}$ with $\alpha+\sigma \in R$. Recall that $\dot{\Pi}=\left\{\alpha_{1}, \ldots, \alpha_{\ell}\right\}$ is a basis for $\dot{R}$. Without loss of generality, we may assume that $\left(\alpha_{1}, \alpha_{2}\right) \in \dot{R}_{s h} \times \dot{R}_{l g}$ and $\left(\alpha_{1}, \alpha_{2}\right) \neq 0$. Let $\alpha \in R^{\times}$and $\sigma \in \mathcal{V}^{0}$ so that $\alpha+\sigma \in R$. Then by (1.9) we have $\alpha=\dot{\alpha}+\delta$ and $\alpha+\sigma=\dot{\alpha}+\delta^{\prime}$, where $\dot{\alpha} \in \dot{R}_{s h} \uplus \dot{R}_{l g}$ and $\delta, \delta^{\prime} \in \mathcal{V}^{0}$ and so from (1.9), (1.12) and the facts that $\dot{R}_{s h}=\dot{\mathcal{W}} \alpha_{1}, \dot{R}_{l g}=\dot{\mathcal{W}} \alpha_{2}, 2 \Lambda_{1}=2 \Sigma_{r=1}^{t} \mathbb{Z} \sigma_{r}$ and $2 \Lambda_{2}=2 \Sigma_{r=t+1}^{\nu} \mathbb{Z} \sigma_{r}$, we get

$$
\begin{gathered}
\alpha= \begin{cases}\dot{w}\left(\alpha_{1}\right)+\tau_{J}+\sum_{r=1}^{\nu} n_{r} b_{1}(r) \sigma_{r}, & \text { if } \alpha \in \dot{R}_{s h}+S, \\
\dot{w}\left(\alpha_{2}\right)+\tau_{J}+\sum_{r=1}^{\nu} n_{r} b_{2}(r) k_{r} \sigma_{r}, & \text { if } \alpha \in \dot{R}_{l g}+L,\end{cases} \\
\alpha+\sigma= \begin{cases}\dot{w}\left(\alpha_{1}\right)+\tau_{J^{\prime}}+\sum_{r=1}^{\nu} n_{r}^{\prime} b_{1}(r) \sigma_{r}, & \text { if } \alpha \in \dot{R}_{s h}+S, \\
\dot{w}\left(\alpha_{2}\right)+\tau_{J^{\prime}}+\sum_{r=1}^{\nu} n_{r}^{\prime} b_{2}(r) k_{r} \sigma_{r}, & \text { if } \alpha \in \dot{R}_{l g}+L,\end{cases}
\end{gathered}
$$

where $\dot{\alpha}=\dot{w}\left(\alpha_{j}\right)$ for some $\dot{w} \in \dot{\mathcal{W}}$ and $j \in\{1,2\}, J, J^{\prime} \in \operatorname{supp}_{\mathcal{B}_{j}}\left(S_{j}\right), n_{r}, n_{r}^{\prime} \in$ $\mathbb{Z}$, and

$$
b_{1}(r):=\left\{\begin{array}{l}
2, \text { if } 1 \leq r \leq t, \\
1, \text { if } t<r \leq \nu,
\end{array} \quad \text { and } \quad b_{2}(r):=\left\{\begin{array}{l}
1, \text { if } 1 \leq r \leq t \\
2, \text { if } t<r \leq \nu
\end{array}\right.\right.
$$


We now set

$$
\begin{aligned}
w_{1} & :=\prod_{r=1}^{t} t_{1, r}^{n_{r}} \prod_{r=t+1}^{\nu} t_{2, r}^{-n_{r}}, \quad w_{1}^{\prime}:=\prod_{r=1}^{t} t_{1, r}^{n_{r}^{\prime}} \prod_{r=t+1}^{\nu} t_{2, r}^{-n_{r}^{\prime}} \\
w_{2} & :=\prod_{r=1}^{t} t_{1, r}^{-n_{r}} \prod_{r=t+1}^{\nu} t_{2, r}^{n_{r}} \quad \text { and } \quad w_{2}^{\prime}:=\prod_{r=1}^{t} t_{1, r}^{-n_{r}^{\prime}} \prod_{r=t+1}^{\nu} t_{2, r}^{n_{r}^{\prime}} .
\end{aligned}
$$

Then from the facts that $\left(\alpha_{2}, \alpha_{1}^{\vee}\right)=-k$ and $\left(\alpha_{1}, \alpha_{2}^{\vee}\right)=-1$ and using (2.11), (2.27), (2.28) and (2.29) we get $\alpha=\dot{w} w_{j}\left(\alpha_{j}+\tau_{J}\right)$ and $\alpha+\sigma=\dot{w} w_{j}^{\prime}\left(\alpha_{j}+\tau_{J^{\prime}}\right)$, $j=1,2$ and so we have

$$
\begin{aligned}
w_{\alpha} & =w_{\dot{w} w_{j}\left(\alpha_{j}+\tau_{J}\right)}=\left(\dot{w} w_{j}\right) w_{\alpha_{j}+\tau_{J}}\left(\dot{w} w_{j}\right)^{-1}=\dot{w} w_{j} w_{\alpha_{j}+\tau_{J}} w_{j}^{-1} \dot{w}^{-1} \\
& =\dot{w} w_{j} w_{\alpha_{j}}\left(w_{\alpha_{j}} w_{\alpha_{j}+\tau_{J}} \prod_{r \in J} t_{j, r}\right)\left(\prod_{r \in J} t_{j, r}\right)^{-1} w_{j}^{-1} \dot{w}^{-1} \\
& =\dot{w} w_{j} w_{\alpha_{j}} z_{J}\left(\prod_{r \in J} t_{j, r}\right)^{-1} w_{j}^{-1} \dot{w}^{-1} \quad \text { (by Lemma 2.3(i)) }
\end{aligned}
$$

and

$$
\begin{aligned}
w_{\alpha+\sigma} & =w_{\dot{w} w_{j}^{\prime}\left(\alpha_{j}+\tau_{J^{\prime}}\right)}=\left(\dot{w} w_{j}^{\prime}\right) w_{\alpha_{j}+\tau_{J^{\prime}}}\left(\dot{w} w_{j}^{\prime}\right)^{-1} \quad(\text { by }(2.2)) \\
& =\dot{w} w_{j}^{\prime} w_{\alpha_{j}+\tau_{J^{\prime}}} w_{j}^{\prime-1} \dot{w}^{-1} \\
& =\dot{w} w_{j}^{\prime} w_{\alpha_{j}}\left(w_{\alpha_{j}} w_{\alpha_{j}+\tau_{J^{\prime}}} \prod_{r \in J^{\prime}} t_{j, r}\right)\left(\prod_{r \in J^{\prime}} t_{j, r}\right)^{-1} w_{j}^{\prime-1} \dot{w}^{-1} \\
& =\dot{w} w_{j}^{\prime} w_{\alpha_{j}} z_{J^{\prime}}\left(\prod_{r \in J^{\prime}} t_{j, r}\right)^{-1} w_{j}^{\prime-1} \dot{w}^{-1} \quad(\text { by Lemma } 2.3(\mathrm{i})) .
\end{aligned}
$$

Thus

$$
\begin{aligned}
w_{\alpha+\sigma} w_{\alpha}= & \left(\dot{w} w_{j}^{\prime} w_{\alpha_{j}} z_{J^{\prime}}\left(\prod_{r \in J^{\prime}} t_{j, r}\right)^{-1} w_{j}^{-1} \dot{w}^{-1}\right) \\
& \times\left(\dot{w} w_{j} w_{\alpha_{j}} z_{J}\left(\prod_{r \in J} t_{j, r}\right)^{-1} w_{j}^{-1} \dot{w}^{-1}\right) .
\end{aligned}
$$

Then from the facts that $w_{j}, w_{j}^{\prime} \in T$ and $\dot{w} \in T^{\prime}, j=1,2$, it follows that $w_{\alpha+\sigma} w_{\alpha} \in T$ and $w_{\alpha} \in T^{\prime}$.

(iii) By Lemma 1.3(i) and Corollary 1.1 of [AzS2], (iii) holds (see also [Az1, Proposition 3.27]).

(iv) This is an immediate consequence of (i)-(iii), Lemma 2.5(i) and the fact that $\dot{\mathcal{W}}$ is a finite group. 
(v) Let $w \in \mathcal{W}$. By (i), (2.14) and Lemma 2.5, $w$ has an expression of the given form. Now let $w\left(\dot{w}^{\prime},\left\{n_{i, r}^{\prime}\right\}, u_{1}^{\prime},\left\{m_{r, s}^{\prime}\right\}, u_{2}^{\prime}\right)$ be another expression of $w$ in the form (2.26). Then from (i) and (ii) we have $\dot{w}=\dot{w}^{\prime}$ and

$$
\prod_{r=1}^{\nu} \prod_{i=1}^{\ell} t_{i, r}^{n_{i, r}} u_{1} \prod_{1 \leq r \leq t<s \leq \nu} z_{\{r, s\}}^{m_{r, s}} u_{2}=\prod_{r=1}^{\nu} \prod_{i=1}^{\ell} t_{i, r}^{n_{i, r}^{\prime}} u_{1 \leq r \leq t<s \leq \nu}^{\prime} \prod_{1 \leq t, j}^{m_{r, s}^{\prime}} u_{2}^{\prime}
$$

By the way $z_{J}$ 's are defined (see (2.13)) and the fact that $J \subseteq\{1, \ldots, t\}$ and $J^{\prime} \subseteq\{t+1, \ldots, \nu\}$ for all $\left(J, J^{\prime}\right) \in \operatorname{supp}_{\mathcal{B}_{1}}\left(S_{1}\right) \times \operatorname{supp}_{\mathcal{B}_{2}}\left(S_{2}\right)$, the elements $u_{1}, u_{1}^{\prime}, u_{2}$ and $u_{2}^{\prime}$ can be written in the forms

$$
u_{1}=\prod_{1 \leq r<s \leq t} c_{r, s}^{m_{r, s}}, \quad u_{1}^{\prime}=\prod_{1 \leq r<s \leq t} c_{r, s}^{m_{r, s}^{\prime}}, \quad u_{2}=\prod_{t<r<s \leq \nu} c_{r, s}^{m_{r, s}}, \quad \text { and } \quad u_{2}^{\prime}=\prod_{t<r<s \leq \nu} c_{r, s}^{m_{r, s}^{\prime}},
$$

where $m_{r, s}, m_{r, s}^{\prime} \in \mathbb{Z}$. Then from (2.8), (2.30) and (2.13), it follows that

$$
\prod_{r=1}^{\nu} \prod_{i=1}^{\ell} T_{\alpha_{i}^{\vee}}^{n_{i, r} k_{i, r} \sigma_{r}} \prod_{1 \leq r<s \leq \nu} T_{\sigma_{r}}^{m_{r, s} k^{-1} \sigma_{s}}=\prod_{r=1}^{\nu} \prod_{i=1}^{\ell} T_{\alpha_{i}^{\vee}}^{n_{i, r}^{\prime} k_{i, r} \sigma_{r}} \prod_{1 \leq r<s \leq \nu} T_{\sigma_{r}}^{m_{r, s}^{\prime} k^{-1} \sigma_{s}}
$$

Now using [AzS2, Lemma 1.6] we have $n_{i, r}=n_{i, r}^{\prime}$, for all $1 \leq i \leq \ell, 1 \leq r \leq \nu$ and $m_{r, s}=m_{r, s}^{\prime}$ for all $1 \leq r<s \leq \nu$ and so $u_{1}=u_{1}^{\prime}$ and $u_{2}=u_{2}^{\prime}$.

(vi) Let $F$ be the group on right hand side of the statement. By Lemma 2.3 , it is clear that $F \subseteq Z(\mathcal{W})$. To see the reverse inclusion, let $w \in Z(\mathcal{W})$. By $(\mathrm{v}), w$ has an expression of the form

$$
w=\dot{w} \prod_{r=1}^{\nu} \prod_{i=1}^{\ell} t_{i, r}^{n_{i, r}} u, \quad\left(\dot{w} \in \dot{\mathcal{W}}, \quad n_{i, r} \in \mathbb{Z}, \quad u \in F\right) .
$$

We must show that $\dot{w}=1$ and $n_{i, r}=0$ for any $1 \leq i \leq \ell$ and $1 \leq r \leq \nu$. For any $1 \leq i \leq \ell$, set $\beta_{i}:=w\left(\alpha_{i}^{\vee}\right)-\alpha_{i}^{\vee}$. Then from the fact that $t_{i, r} w^{-1} t_{i, r}^{-1}=1$ for any $1 \leq i \leq \ell, 1 \leq r \leq \nu$, we get

$$
\begin{aligned}
\lambda_{r} & =w t_{i, r} w^{-1} t_{i, r}^{-1}\left(\lambda_{r}\right) \\
& =w T_{\alpha_{i}^{\vee}}^{k_{i, r} \sigma_{r}} w^{-1} T_{-\alpha_{i}^{\vee}}^{k_{i, r} \sigma_{r}}\left(\lambda_{r}\right) \quad(\text { by }(2.8)) \\
& =T_{w\left(\alpha_{i}^{\vee}\right)}^{k_{i, r} \sigma_{r}} T_{-\alpha_{i}^{\vee}}^{k_{i, r} \sigma_{r}}\left(\lambda_{r}\right) \quad(\text { by Lemma } 2.1(\mathrm{vi})) \\
& =T_{w\left(\alpha_{i}^{\vee}\right)-\alpha_{i}^{\vee}}^{k_{i, r} \sigma_{r}}\left(\lambda_{r}\right)=T_{\beta_{i}}^{k_{i, r} \sigma_{r}}\left(\lambda_{r}\right) \quad(\text { by Lemma } 2.1(\mathrm{iii})) \\
& \left.=\lambda_{r}-\beta_{i}+\left(\beta_{i}, \lambda_{r}\right) k_{i, r} \sigma_{r}-\frac{\left(\beta_{i}, \beta_{i}\right)}{2}\left(\beta_{i}, \lambda_{r}\right) k_{i, r}^{2} \sigma_{r} \quad \text { (by }(2.1) \text { and }(2.7)\right) .
\end{aligned}
$$


Hence $\beta_{i}=\left[\left(\beta_{i}, \lambda_{r}\right) k_{i, r}-k_{i, r}^{2}\left(\beta_{i}, \beta_{i}\right) / 2\right] \sigma_{r} \in \mathcal{V}^{0}$ and so by the fact that $\dot{w}^{-1} w\left(\alpha_{i}^{\vee}\right)$ $=\alpha_{i}^{\vee}+\delta$ for some $\delta \in \mathcal{V}^{0}$, we have

$$
\begin{aligned}
\dot{w}^{-1}\left(\alpha_{i}^{\vee}\right)-\alpha_{i}^{\vee} & =-\dot{w}^{-1} w\left(\alpha_{i}^{\vee}\right)+\dot{w}^{-1}\left(\alpha_{i}^{\vee}\right)-\alpha_{i}^{\vee}+\dot{w}^{-1} w\left(\alpha_{i}^{\vee}\right) \\
& =\dot{w}^{-1}\left(-w\left(\alpha_{i}^{\vee}\right)+\alpha_{i}^{\vee}\right)+\delta \\
& =\dot{w}^{-1}\left(-\beta_{i}\right)+\delta=-\beta_{i}+\delta \in \mathcal{V}^{0} .
\end{aligned}
$$

This gives $\dot{w}\left(\alpha_{i}^{\vee}\right)=\alpha_{i}^{\vee}$ for all $1 \leq i \leq \ell$ and so $\dot{w}=1$. So to complete the proof of (vi) it remains to show that $n_{i, r}=0$ for $1 \leq i \leq \ell$ and $1 \leq r \leq \nu$. First, let $\nu=1$. Set $\dot{\alpha}:=\Sigma_{i=1}^{\ell} n_{i, 1} \alpha_{i}^{\vee}$ and $\dot{\beta}:=\dot{\alpha}-w_{\alpha_{j}}(\dot{\alpha})=\left(\dot{\alpha}, \alpha_{j}^{\vee}\right) \alpha_{j} \in \dot{V}$. Then using the facts that $w=\prod_{i=1}^{\ell} t_{i, 1}^{n_{i, 1}} \in Z(\mathcal{W})$ (see (2.31)) and $F=\{1\}$ we have (for any $1 \leq j \leq \ell$ )

$$
\begin{aligned}
\lambda_{1} & =w w_{\alpha_{j}} w^{-1} w_{\alpha_{j}}\left(\lambda_{1}\right) \\
& =\prod_{i=1}^{\ell} t_{i, 1}^{n_{i, 1}} w_{\alpha_{j}}\left(\prod_{i=1}^{\ell} t_{i, 1}^{n_{i, 1}}\right)^{-1} w_{\alpha_{j}}\left(\lambda_{1}\right) \\
& =\prod_{i=1}^{\ell}\left(T_{\alpha_{i}^{\vee}}^{k_{i, 1} \sigma_{1}}\right)^{n_{i, 1}} w_{\alpha_{j}}\left(\prod_{i=1}^{\ell} T_{\alpha_{i}^{\vee}}^{k_{i, 1} \sigma_{1}}\right)^{-n_{i, 1}} w_{\alpha_{j}}\left(\lambda_{1}\right) \quad(\text { by }(2.8)) \\
& =T_{\Sigma_{i=1}^{\ell} k_{i, 1} \sigma_{1}}^{k_{i, 1} \alpha_{i}^{\vee}} w_{\alpha_{j}}\left(T_{\Sigma_{i=1}^{\prime} n_{i, 1} \alpha_{i}^{\vee}}^{k_{i, 1} \sigma_{1}}\right)^{-1} w_{\alpha_{j}}\left(\lambda_{1}\right) \quad(\text { by Lemma } 2.1(\mathrm{iii})) \\
& =T_{\dot{\alpha}}^{k_{i, 1} \sigma_{1}} w_{\alpha_{j}}\left(T_{\dot{\alpha}}^{k_{i, 1} \sigma_{1}}\right)^{-1} w_{\alpha_{j}}\left(\lambda_{1}\right)=T_{\dot{\alpha}}^{k_{i, 1} \sigma_{1}} T_{-w_{\alpha_{j}}(\dot{\alpha})}^{k_{i, 1} \sigma_{1}}\left(\lambda_{1}\right) \\
& =T_{\dot{\beta}}^{k_{i, 1} \sigma_{1}}\left(\lambda_{1}\right) \quad(\text { by Lemma } 2.1(\mathrm{iii})) \\
& \left.=\lambda_{1}+\left(\dot{\beta}, \lambda_{1}\right) k_{i, 1} \sigma_{1}-\left(k_{i, 1} \sigma_{1}, \lambda_{1}\right) \dot{\beta}-\frac{(\dot{\beta}, \dot{\beta})}{2}\left(k_{i, 1} \sigma_{1}, \lambda_{1}\right) k_{i, 1} \sigma_{1} \quad \text { (by }(2.7)\right) \\
& =\lambda_{1}-k_{i, 1} \dot{\beta}-\frac{(\dot{\beta}, \dot{\beta})}{2} k_{i, 1}^{2} \sigma_{1} \quad(\text { by }(2.1)) .
\end{aligned}
$$

Then $\dot{\beta}=\frac{(\dot{\beta}, \dot{\beta})}{2} k_{i, 1} \sigma_{1} \in \dot{\mathcal{V}} \cap \mathcal{V}^{0}=\{0\}$ and so $\dot{\beta}=\left(\dot{\alpha}, \alpha_{j}^{\vee}\right) \alpha_{j}=0$ for all $1 \leq j \leq \ell$. Since the restriction of the form $(\cdot, \cdot)$ to $\dot{V}=\sum_{i=1}^{\ell} \mathbb{R} \alpha_{i}$ is positive definite we get $n_{i, r}=0$ for all $1 \leq i \leq \ell$ and $1 \leq r \leq \nu$. Next, let $\nu \geq 2$. Then from the fact that $\dot{w}=1$ we have (for any $1 \leq j \leq \ell, 1 \leq s \leq \nu$ )

$$
\begin{aligned}
1 & =\left[w, t_{j, s}\right] \\
& =\left[\prod_{r=1}^{\nu} \prod_{i=1}^{\ell} t_{i, r}^{n_{i, r}} u, t_{j, s}\right] \quad(\text { by }(2.31)) \\
& =\prod_{r=1}^{\nu} \prod_{i=1}^{\ell}\left[t_{i, r}^{n_{i, r}}, t_{j, s}\right] \quad \text { (by (iii) and Lemma 2.1(v)) }
\end{aligned}
$$




$$
\begin{aligned}
& =\prod_{r=1}^{s-1} \prod_{i=1}^{\ell}\left[t_{i, r}^{n_{i, r}}, t_{j, s}\right] \prod_{i=1}^{\ell}\left[t_{i, s}^{n_{i, s}}, t_{j, s}\right] \prod_{r=s+1}^{\nu} \prod_{i=1}^{\ell}\left[t_{i, r}^{n_{i, r}}, t_{j, s}\right] \\
& =\prod_{r=1}^{s-1} \prod_{i=1}^{\ell}\left[t_{i, r}^{n_{i, r}}, t_{j, s}\right] \prod_{r=s+1}^{\nu} \prod_{i=1}^{\ell}\left[t_{i, s}, t_{j, r}^{n_{i, r}}\right]^{-1} \\
& =\prod_{r=1}^{s-1} \prod_{i=1}^{\ell} z_{\{r, s\}}^{n_{i, r} \Delta(r, s)^{-1} a_{i, j}(r, s)} \prod_{r=s+1}^{\nu} \prod_{i=1}^{\ell} z_{\{r, s\}}^{-n_{i, r} \Delta(s, r)^{-1} a_{i, j}(s, r)} \text { (by Lemma 2.5(i)). }
\end{aligned}
$$

Since $\left\langle z_{\{r, s\}} \mid 1 \leq r<s \leq \nu\right\rangle$ is a free abelian group of rank $\nu(\nu-1) / 2$ on generators $z_{\{r, s\}}, 1 \leq r<s \leq \nu$ (see Lemma 2.2(i)) from the way $a_{i, j}(r, s)$ 's are defined (see $(2.15))$ we have $n_{i, r}\left(\alpha_{i}, \alpha_{j}\right)=0$ for all $1 \leq j \leq \ell$ and so from the fact that $\left(\alpha_{i}, \alpha_{i}\right) \neq 0$, it follows that $n_{i, r}=0$ for all $1 \leq i \leq \ell$ and $1 \leq r \leq \nu$.

(vii) From (i) and Lemma 1.1(i) we have $\mathcal{H}=\left\langle t_{i, 1} \mid 1 \leq i \leq \ell\right\rangle$. So by Lemma 2.5(i) and the fact that $z_{\{1,1\}}=1,(\mathrm{v}), \mathcal{H}$ is a free abelian group of rank $\ell$ on generators $t_{i, 1}, 1 \leq i \leq \ell$. Also by (vi) it is clear that $Z(\mathcal{W})=\{1\}$.

(viii) From (vi) and its proof, it follows that

$$
\begin{aligned}
Z(\mathcal{W})=Z(\mathcal{H}) & =\left\langle z_{\{r, s\}}, z_{J} \mid 1 \leq r<s \leq \nu, J \in \mathcal{J}_{\mathcal{B}_{1}}\left(S_{1}\right) \cup \mathcal{J}_{\mathcal{B}_{2}}\left(S_{2}\right)\right\rangle \\
& =\left\langle z_{J} \mid J \subseteq\{1, \ldots, \nu\}\right\rangle
\end{aligned}
$$

and so by Lemma 2.2(ii), $Z(\mathcal{H})$ is a free abelian group of rank $\nu(\nu-1) / 2$.

\section{$\S 3 . \quad$ A Finite Presentation for Extended Affine Weyl Group $\mathcal{W}$}

We keep the notation as in the previous sections. In particular, $R$ is a reduced non-simply laced extended affine root system of type $X_{\ell}$ of twist number $t$ and nullity $\nu$ of the form (1.9) and $\mathcal{W}$ is its extended affine group. Also $\dot{\Pi}=\left\{\alpha_{1}, \ldots, \alpha_{\ell}\right\}$ is a basis of $\dot{R}$. Let $\mathcal{B}_{1}=\left\{\sigma_{1}, \ldots, \sigma_{t}\right\}$ and $\mathcal{B}_{2}=$ $\left\{\sigma_{t+1}, \ldots, \sigma_{\nu}\right\}$ be two bases for semilattices $S_{1}$ and $S_{2}$, respectively. Using the Coxeter presentation for the finite Weyl group $\dot{\mathcal{W}}$ and results from Section 2 we obtain a finite presentation for $\mathcal{W}$.

Let $A=\left(a_{i, j}\right)_{1 \leq i, j \leq \ell}$ be the Cartan matrix of type $X_{\ell}$, that is $a_{i, j}=$ $\left(\alpha_{i}, \alpha_{j}^{\vee}\right), 1 \leq i, j \in \leq \ell$.

We recall from [K, Proposition 3.13] that $\dot{\mathcal{W}}$ is a Coxeter group with generators $w_{\alpha_{1}}, \ldots, w_{\alpha_{\ell}}$ and relations

$$
w_{\alpha_{i}}^{2}=1 \quad \text { and } \quad\left(w_{\alpha_{i}} w_{\alpha_{j}}\right)^{m_{i, j}}=1 \quad(i \neq j)
$$


where $m_{i, j}$ 's are given in terms of the Cartan matrix by the following table:

\begin{tabular}{|c|r|r|r|r|}
\hline$a_{i, j} a_{j, i}$ & 0 & 1 & 2 & 3 \\
\hline$m_{i, j}$ & 2 & 3 & 4 & 6 \\
\hline
\end{tabular}

Theorem 3.1. Let $R=R\left(X_{\ell}, S_{1}, S_{2}\right)$ be a reduced non-simply laced extended affine root system of rank $\ell$, nullity $\nu$, twist number $t$ and of the form (1.9). Then the extended affine Weyl group $\mathcal{W}$ of $R$ is isomorphic to the group $\widehat{\mathcal{W}}$ defined by generators

$$
\left\{\begin{array}{l}
\hat{w}_{i}, \hat{t}_{i, r}, 1 \leq i \leq \ell, \quad 1 \leq r \leq \nu, \\
\hat{z}_{r, s}, \quad 1 \leq r<s \leq \nu, \\
\hat{z}_{J}, \quad J \in \mathcal{J}_{\mathcal{B}_{1}}\left(S_{1}\right) \cup \mathcal{J}_{\mathcal{B}_{2}}\left(S_{2}\right),
\end{array}\right.
$$

$\left(\mathcal{J}_{\mathcal{B}_{1}}\left(S_{1}\right)\right.$ and $\mathcal{J}_{\mathcal{B}_{2}}\left(S_{2}\right)$ are defined by $\left.(1.3)\right)$ and relations

(I) $\hat{w}_{i}^{2}=1$ and $\left(\hat{w}_{i} \hat{w}_{j}\right)^{m_{i, j}}=1,(i \neq j),\left(m_{i, j}\right.$ 's are given by table $\left.(3.2)\right)$

(II) $\hat{w}_{i} \hat{t}_{j, r} \hat{w}_{i}=\hat{t}_{j, r} \hat{t}_{i, r}^{-a_{i, j}(r)},\left(a_{i, j}(r)\right.$ 's are given by $\left.(2.16)\right)$,

(III) $\left[\hat{t}_{i, r}, \hat{t}_{j, s}\right]=\hat{z}_{r, s}^{\Delta(r, s)^{-1} a_{i, j}(r, s)},\left(\Delta(r, s)^{\prime}\right.$ 's and $a_{i, j}(r, s)^{\prime}$ 's are given by (2.12) and (2.17)),

$(\mathbf{I V})\left\{\begin{array}{l}{\left[\hat{w}_{i}, \hat{z}_{r, s}\right]=\left[\hat{w}_{i}, \hat{z}_{J}\right]=\left[\hat{t}_{i, r}, \hat{z}_{J}\right]=\left[\hat{t}_{i, r}, \hat{z}_{r, s}\right]=1,} \\ {\left[\hat{t}_{i, r}, \hat{t}_{j, r}\right]=\left[\hat{z}_{r, s}, \hat{z}_{r^{\prime}, s^{\prime}}\right]=\left[\hat{z}_{r, s}, \hat{z}_{J}\right]=\left[\hat{z}_{J}, \hat{z}_{J^{\prime}}\right]=1,}\end{array}\right.$

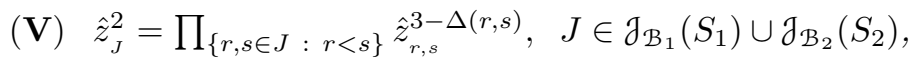

(VI.1) $\prod_{1 \leq r<s \leq t} \hat{z}_{r, s}^{m_{r, s}} \prod_{J \in \mathcal{I}_{\mathcal{B}_{1}}\left(S_{1}\right)} \hat{z}_{J}^{\epsilon_{J}}=1$, for $m_{r, s} \in \mathbb{Z}$ and $\epsilon_{J} \in\{0,1\}$ satisfying

$$
\begin{gathered}
m_{r, s}=-\Delta(r, s)^{-1} \sum_{J \in \mathcal{J}_{\mathcal{B}_{1}}\left(S_{1}\right)} \chi_{J}(r, s) \epsilon_{J} \quad \text { for all } 1 \leq r<s \leq t, \text { where } \\
\chi_{J}(r, s)=\left\{\begin{array}{l}
1, \text { if }\{r, s\} \subsetneq J, \\
0, \text { otherwise, }
\end{array}\right.
\end{gathered}
$$


(VI.2) $\prod_{t<r<s \leq \nu} \hat{z}_{r, s}^{n_{r, s}} \prod_{J \in \mathcal{J}_{\mathcal{B}_{2}\left(S_{2}\right)}} \hat{z}_{J}^{\varepsilon_{J}}=1$, for $n_{r, s} \in \mathbb{Z}$ and $\epsilon_{J} \in\{0,1\}$ satisfying

$$
n_{r, s}=-\Delta(r, s)^{-1} \sum_{J \in \mathcal{J}_{\mathcal{B}_{2}}\left(S_{2}\right)} \chi_{J}(r, s) \epsilon_{J} \quad \text { for all } t<r<s \leq \nu .
$$

Moreover, $Z(\widehat{\mathcal{W}})=\left\langle\hat{z}_{r, s}, \hat{z}_{J} \mid 1 \leq r<s \leq \nu, \quad J \in \mathcal{J}_{\mathcal{B}_{1}}\left(S_{1}\right) \cup \mathcal{J}_{\mathcal{B}_{2}}\left(S_{2}\right)\right\rangle$.

Proof. From parts (ii) and (vi) of Proposition 2.1, Lemma 2.5(i)-(ii) and (3.1), it follows that the assignment $\hat{w}_{i} \longmapsto w_{\alpha_{i}}, \hat{t}_{i, r} \longmapsto t_{i, r}, \hat{z}_{r, s} \longmapsto z_{\{r, s\}}$ and $\hat{z}_{J} \longmapsto z_{J}$, induces a unique epimorphism $\psi: \widehat{\mathcal{W}} \longrightarrow \mathcal{W}$. Consider the subgroup

$$
\widehat{\dot{\mathcal{W}}}:=\left\langle\hat{w}_{i} \mid 1 \leq i \leq \ell\right\rangle
$$

of $\widehat{\mathcal{W}}$. By (3.1), the restriction of $\psi$ to $\widehat{\dot{\mathcal{W}}}$ induces the isomorphism

$$
\widehat{\dot{\mathcal{W}}} \stackrel{\Downarrow}{\cong} \text {. }
$$

We now show that $\psi$ is injective. Let $\psi(\hat{w})=1$, for some $\hat{w} \in \widehat{\mathcal{W}}$. From the defining relations $\mathbf{V}$, we know that any even power of generators $\hat{z}_{J}$ can be written in terms of generators $\hat{z}_{r, s}, 1 \leq r<s \leq \nu$. Therefore using defining relations $\mathbf{I I}-\mathbf{V}$, we see that $\hat{w}$ can be written in the form:

$$
\hat{w}=\hat{\dot{w}} \prod_{i=1}^{\ell} \prod_{r=1}^{\nu} \hat{t}_{i, r}^{n_{i, r}^{\prime}} \quad \underline{\hat{u}}(\underline{m}, \underline{\epsilon}) \prod_{1 \leq r \leq t<s \leq \nu} \hat{z}_{r, s}^{m_{r, s}^{\prime}} \overline{\hat{u}}(\bar{n}, \bar{\varepsilon}),
$$

where $n_{i, r}^{\prime}, m_{r, s}^{\prime} \in \mathbb{Z},(\underline{m}, \underline{\epsilon})$ and $(\bar{n}, \bar{\varepsilon})$ are pairs of the forms (2.20) and (2.21), respectively and

$$
\underline{\hat{u}}(\underline{m}, \underline{\epsilon}):=\prod_{1 \leq r<s \leq t} \hat{z}_{r, s}^{m_{r, s}} \prod_{J \in \mathcal{J}_{\mathcal{B}_{1}}\left(S_{1}\right)} \hat{z}_{J}^{\epsilon_{J}} \text { and } \overline{\hat{u}}(\bar{n}, \bar{\varepsilon}):=\prod_{t<r<s \leq \nu} \hat{z}_{r, s}^{n_{r, s}} \prod_{J \in \mathcal{J}_{\mathcal{B}_{2}}\left(S_{2}\right)} \hat{z}_{J}^{\varepsilon_{J^{\prime}}} .
$$

Then from (3.5) we have

$$
1=\psi(\hat{w})=\psi(\hat{\dot{w}}) \prod_{i=1}^{\ell} \prod_{r=1}^{\nu} t_{i, r}^{n_{i, r}^{\prime}} \quad \underline{u}(\underline{m}, \underline{\epsilon}) \prod_{1 \leq r \leq t<s \leq \nu} z_{\{r, s\}}^{m_{r, s}^{\prime}} \bar{u}(\bar{n}, \bar{\varepsilon}) .
$$

So from Proposition 2.1(v) and (3.4), it follows that $\hat{\dot{w}}=1$ and $n_{i, r}^{\prime}=0$ for all $i, r ; m_{r, s}^{\prime}=0$ for all $1 \leq r \leq t<s \leq \nu, \underline{u}(\underline{m}, \underline{\epsilon})=1$ and $\bar{u}(\bar{n}, \bar{\varepsilon})=1$. Also by Lemma 2.6 , the pairs $(\underline{m}, \underline{\epsilon})$ and $(\bar{n}, \bar{\varepsilon})$ satisfy $(2.24)$ and (2.25), respectively and so using defining relations VI.1, VI.2 and (3.5) (together with $\hat{\dot{w}}=1$, 
$n_{i, r}=0$ for all $i, r$ and $m_{r, s}=0$ for all $\left.1 \leq r \leq t<s \leq \nu\right)$ we get $\hat{w}=1$. Thus $\psi$ is an isomorphism. Finally, from Proposition 2.1(vi), the way $\psi$ is defined and the fact that $\psi(Z(\widehat{\mathcal{W}}))=Z(\mathcal{W})$ we get

$$
Z(\widehat{\mathcal{W}})=\left\langle\hat{z}_{r, s}, \hat{z}_{J} \mid 1 \leq r<s \leq \nu, \quad J \in \mathcal{J}_{\mathcal{B}_{1}}\left(S_{1}\right) \cup \mathcal{J}_{\mathcal{B}_{2}}\left(S_{2}\right)\right\rangle
$$

and this completes the proof.

Corollary 3.1. Let $R=R\left(X_{\ell}, S_{1}, S_{2}\right)$ be a reduced non-simply laced extended affine root system of rank $\ell$, nullity $\nu$, twist number $t$ and of the form (1.9). If $S_{1}$ and $S_{2}$ are lattices (in particular, for types $F_{4}$ and $G_{2}$ ), then the extended affine Weyl group of $R$ is isomorphic to the group defined by generators

$$
\left\{\begin{array}{l}
\hat{w}_{i}, \quad \hat{t}_{i, r}, 1 \leq i \leq \ell, \quad 1 \leq r \leq \nu \\
\hat{z}_{r, s}, \quad 1 \leq r<s \leq \nu
\end{array}\right.
$$

and relations $\left[\hat{w}_{i}, \hat{z}_{r, s}\right]=\left[\hat{t}_{i, r^{\prime}}, \hat{z}_{r, s}\right]=\left[\hat{t}_{i, r^{\prime}}, \hat{t}_{j, r^{\prime}}\right]=1$, I, II and III, where $\Delta(r, s)=1$ for all $1 \leq r<s \leq \nu$. Moreover, $Z(\widehat{\mathcal{W}})=\left\langle\hat{z}_{r, s} \mid 1 \leq r<s \leq \nu\right\rangle$.

Proof. By Lemma 1.1(i), $\mathcal{J}_{\mathcal{B}_{1}}\left(S_{1}\right)=\mathcal{J}_{\mathcal{B}_{2}}\left(S_{2}\right)=\emptyset$ and so any generator or relation in Theorem 3.1, which is indexed by $\mathcal{J}_{\mathcal{B}_{1}}\left(S_{1}\right)$ or $\mathcal{J}_{\mathcal{B}_{2}}\left(S_{2}\right)$ will be surplus. Now the result follows using the fact that $\Delta(r, s)=1$ for all $1 \leq r<s \leq \nu$.

We note from Lemma 1.1 that if $S$ is a semilattice with $\operatorname{rank}(S) \leq 2$, then $\mathcal{J}_{\mathcal{B}}(S)=\emptyset$. Now this fact together with Theorem 3.1 implies the following two corollaries.

Corollary 3.2 (Affine case). If $R$ is an extended affine root system of type $X_{\ell}$ of nullity $\nu=1$ and twist number $t$, then its extended affine Weyl group is isomorphic to the group $\widehat{\mathcal{W}}$ defined by generators $\hat{w}_{i}, \hat{t}_{i}, 1 \leq i \leq \ell$, and relations $\mathbf{I}, \hat{w}_{i} \hat{t}_{j} \hat{w}_{i}=\hat{t}_{j} \hat{t}_{i}^{-a_{i, j}(1)}$ and $\left[\hat{t}_{i}, \hat{t}_{j}\right]=1,1 \leq i, j \leq \ell$. Moreover, $Z(\widehat{\mathcal{W}})=\langle 1\rangle$.

Corollary 3.3 (Elliptic case). If $R$ is an extended affine root system of type $X_{\ell}$ of nullity $\nu=2$ and twist number $t$ of the form (1.9), then its extended affine Weyl group is isomorphic to the group $\widehat{\mathcal{W}}$ defined by generators $\hat{w}_{i}, \hat{t}_{i, r}, \hat{z}, 1 \leq i \leq \ell, 1 \leq r \leq 2$, and relations $\mathbf{I}, \mathbf{I I},\left[\hat{w}_{i}, \hat{z}\right]=\left[\hat{t}_{i, r}, \hat{z}\right]=$ $\left[\hat{t}_{i, r}, \hat{t}_{j, r}\right]=1$ and $\left[\hat{t}_{i, 1}, \hat{t}_{j, 2}\right]=\hat{z}^{a_{i, j}(1,2) \Delta(1,2)^{-1}}$, where if $X_{\ell}=F_{4}$ or $G_{2}$, then 
$\Delta(1,2)=1$ and for types $B_{\ell}, C_{\ell}, \Delta(1,2)$ is given by

\begin{tabular}{rr|c|c} 
& $t$ & $\left(\operatorname{ind}\left(S_{1}\right), \operatorname{ind}\left(S_{2}\right)\right)$ & $\Delta(1,2)$ \\
\hline 0 & $(0,2)$ & 2 \\
$B_{2}:$ & 0 & $(0,3)$ & 1 \\
1 & $(1,1)$ & 1 \\
2 & $(2,0)$ & 2 \\
2 & $(3,0)$ & 1 \\
\hline
\end{tabular}

\begin{tabular}{r|c|c}
$t$ & $\left(\operatorname{ind}\left(S_{1}\right), \operatorname{ind}\left(S_{2}\right)\right)$ & $\Delta(1,2)$ \\
\cline { 2 - 3 }$B_{\ell}(\ell \geq 3):$ & $(0,3)$ & 1 \\
1 & $(1,1)$ & 1 \\
2 & $(2,0)$ & 2 \\
2 & $(3,0)$ & 1 \\
\hline
\end{tabular}

\begin{tabular}{cc|c|c} 
& $t$ & $\left(\operatorname{ind}\left(S_{1}\right), \operatorname{ind}\left(S_{2}\right)\right)$ & $\Delta(1,2)$ \\
\cline { 2 - 4 }$C_{\ell}(\ell \geq 3):$ & $(0,2)$ & 2 \\
& 0 & $(0,3)$ & 1 \\
& 1 & $(1,1)$ & 1 \\
2 & $(3,0)$ & 1 \\
\hline
\end{tabular}

Moreover, $Z(\widehat{\mathcal{W}})=\langle\hat{z}\rangle$.

Corollary 3.4 (Nullity 3 case). If $R$ is an extended affine root system of type $X_{\ell}$ of nullity 3 and twist number $t$ of the form (1.9), then its extended affine Weyl group is isomorphic to the group $\widehat{\mathcal{W}}$ defined by generators $\hat{w}_{i}, \hat{t}_{i, r}, \hat{z}_{r, s}, 1 \leq i \leq \ell, 1 \leq r \leq 3,1 \leq r<s \leq 3$ and relations $\mathbf{I}$, II, III and $\left[\hat{w}_{i}, \hat{z}_{r, s}\right]=\left[\hat{t}_{i, r}, \hat{z}_{r, s}\right]=\left[\hat{t}_{i, r}, \hat{t}_{j, r}\right]=1$. Moreover, if $X_{\ell}=F_{4}$ or $G_{2}$, then $\Delta(r, s)=1$ for all $1 \leq r<s \leq 3$ and if $X_{\ell}=B_{\ell}$ or $C_{\ell}$, then the integers $\Delta(r, s)$ 's are given by 


\begin{tabular}{|c|c|c|c|c|}
\hline$t$ & $\left(\operatorname{ind}\left(S_{1}\right), \operatorname{ind}\left(S_{2}\right)\right)$ & $\Delta(1,2)$ & $\Delta(1,3)$ & $\Delta(2,3)$ \\
\hline \multirow[t]{5}{*}{0} & $(0,3)$ & 2 & 2 & 2 \\
\hline & $(0,4)$ & 2 & 2 & 1 \\
\hline & $(0,5)$ & 2 & 1 & 1 \\
\hline & $(0,6)$ & 1 & 1 & 1 \\
\hline & $(0,7)$ & 1 & 1 & 1 \\
\hline \multirow[t]{2}{*}{1} & $(1,2)$ & 1 & 1 & 2 \\
\hline & $(1,3)$ & 1 & 1 & 1 \\
\hline \multirow[t]{2}{*}{2} & $(2,1)$ & 2 & 1 & 1 \\
\hline & $(3,1)$ & 1 & 1 & 1 \\
\hline \multirow[t]{5}{*}{3} & $\overline{(3,0)}$ & 2 & 2 & 2 \\
\hline & $(4,0)$ & 1 & 2 & 2 \\
\hline & $(5,0)$ & 1 & 1 & 2 \\
\hline & $(6,0)$ & 1 & 1 & 1 \\
\hline & $(7,0)$ & 1 & 1 & 1 \\
\hline
\end{tabular}

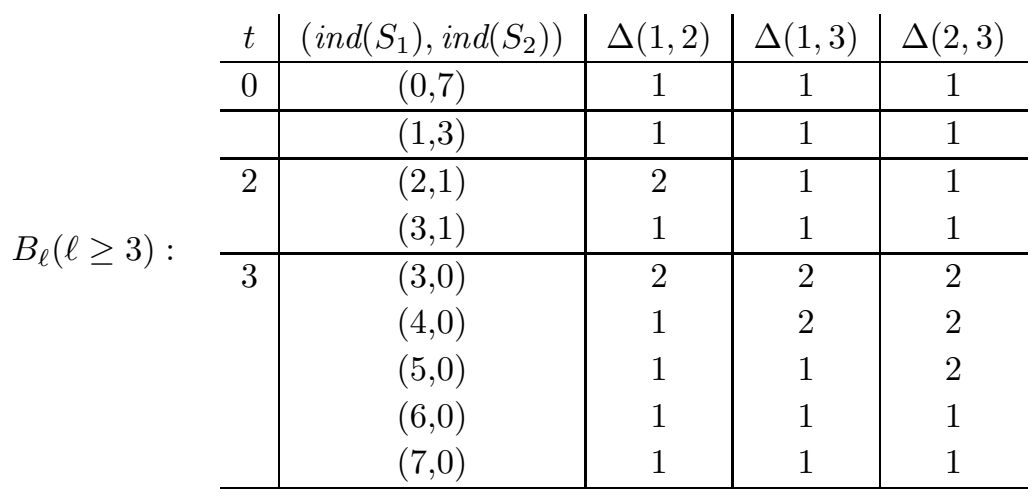

\begin{tabular}{c|c|c|c|c|c} 
& $t$ & $\left(\operatorname{ind}\left(S_{1}\right), \operatorname{ind}\left(S_{2}\right)\right)$ & $\Delta(1,2)$ & $\Delta(1,3)$ & $\Delta(2,3)$ \\
\hline \multirow{5}{*}{$C_{\ell}(\ell \geq 3):$} & $(0,3)$ & 2 & 2 & 2 \\
& & $(0,4)$ & 2 & 2 & 1 \\
& & $(0,5)$ & 2 & 1 & 1 \\
& & $(0,6)$ & 1 & 1 & 1 \\
& & $(0,7)$ & 1 & 1 & 1 \\
\hline \multirow{2}{*}{1} & $(1,2)$ & 1 & 1 & 2 \\
& $(1,3)$ & 1 & 1 & 1 \\
\hline 2 & $(3,1)$ & 1 & 1 & 1 \\
\hline & & $(7,0)$ & 1 & 1 & 1 \\
\hline
\end{tabular}


Moreover, $Z(\widehat{\mathcal{W}})=\left\langle\hat{z}_{1,2}, \hat{z}_{1,3}, \hat{z}_{2,3}\right\rangle$.

Proof. If $X_{\ell}=F_{4}$ or $G_{2}$. Then by Corollary 3.1, the result holds. Next, let $X_{\ell}=B_{\ell \geq 3}$ (resp, $C_{\ell \geq 3}$ ). Then according to [AABGP, Propositions 4.9 and 4.17] and Lemma 1.1, $R$ is isomorphic to an extended affine root system $R^{\prime}=R\left(X_{\ell}, S_{1}^{\prime}, S_{2}^{\prime}\right)$ of the form (1.9) where $\operatorname{supp}_{\mathcal{B}_{1}}\left(S_{1}^{\prime}\right)\left(\operatorname{resp} . \operatorname{supp}_{\mathcal{B}_{2}}\left(S_{2}^{\prime}\right)\right)$ is given by Table 1.4 (see Lemma 1.1(ii)) and $S_{2}^{\prime}$ (resp. $S_{1}^{\prime}$ ) is a lattice. So without loss of generality, we can assume that $S_{1}=S_{1}^{\prime}$ and $S_{2}=S_{2}^{\prime}$. Then by Theorem 3.1 and (2.12), the result holds. Finally, let $X_{\ell}=B_{2}$. Then again we may assume that $\operatorname{supp}_{\mathcal{B}_{1}}\left(S_{1}\right)$ and $\operatorname{supp}_{\mathcal{B}_{2}}\left(S_{2}\right)$ are given by Table 1.4. Now the same argument as in the previous case works also in this case.

Remark 2. (i) If $S_{2}$ (resp. $S_{1}$ ) is a lattice (in particular for type $B_{\ell \geq 3}$ (resp. $\left.C_{\ell \geq 3}\right)$ ), then any generator or relation in Theorem 3.1, which is indexed by $\mathcal{J}_{\mathcal{B}_{2}}\left(S_{2}\right)$ (resp. $\mathcal{J}_{\mathcal{B}_{1}}\left(S_{1}\right)$ ) (see Lemma 1.1(i)) will be surplus. Moreover, $\Delta(r, s)=1$ for all $t+1 \leq r<s \leq \nu$ (resp. $1 \leq r<s \leq t)$ and $1 \leq r \leq t<s \leq \nu$.

(ii) If one of the following conditions holds:

(a) $X_{\ell}=B_{\ell}(\ell \geq 3)$ and $t \leq 3$,

(b) $X_{\ell}=B_{2}, t \leq 3$ and $\nu-t \leq 3$,

(c) $X_{\ell}=C_{\ell}(\ell \geq 3)$ and $\nu-t \leq 3$.

Then using Table 1.4, we may choose $S_{1}$ and $S_{2}$ (and $\mathcal{B}_{1}$ and $\mathcal{B}_{2}$ ) such that $J_{\mathcal{B}_{1}}\left(S_{1}\right)=\mathcal{J}_{\mathcal{B}_{1}}\left(S_{1}\right)=\emptyset$ (see Lemma 1.1) and so any generator or relation in Theorem 3.1, which is indexed by $\mathcal{J}_{\mathcal{B}_{2}}\left(S_{2}\right) \cup \mathcal{J}_{\mathcal{B}_{1}}\left(S_{1}\right)$ will be surplus. Also the integers $\Delta(r, s)$ 's are easily computable using Lemma 1.1(ii) and [AABGP, Propositions 4.9 and 4.17] (see the proof of Corollary 3.4).

(iii) Using an argument analogous to the proof of Theorem 3.1 we can prove that the generators $\hat{t}_{i, r}, \hat{z}_{r, s}$ and $\hat{z}_{J}$ together with relations III, V, VI.j, $j=1,2$ and $\left[\hat{t}_{i, r}, \hat{z}_{J}\right]=\left[\hat{t}_{i, r}, \hat{z}_{r, s}\right]=\left[\hat{t}_{i, r}, \hat{t}_{j, r}\right]=\left[\hat{z}_{r, s}, \hat{z}_{r^{\prime}, s^{\prime}}\right]=\left[\hat{z}_{r, s}, \hat{z}_{J}\right]=\left[\hat{z}_{J}, \hat{z}_{J^{\prime}}\right]=1$ present the Heisenberg-like group $\mathcal{H}$.

\section{$\S 4 . \quad$ Examples}

This section contains some examples in which we have computed the terms appearing in our presentation of an extended affine Weyl group (Theorem 3.1). In Example 1, we compute the supporting class of a semilattice $S$ of rank 4, the supporting function $\delta(r, s)$ and the set $\mathcal{J}_{\mathcal{B}}(S)$. In Examples 2 and 3, we consider the extended affine Weyl groups of two extended affine root systems of type $B_{2}$, one with nullity 3 and the other with nullity 7 . The supporting 
class, the sets $\mathcal{J}_{\mathcal{B}_{i}}\left(S_{i}\right)$ 's, the integers $k_{r}$ 's, $k_{i, r}$ 's and $\Delta(r, s)$ 's are computed. Moreover the presentation stated in Theorem 3.1 is explicitly given for these particular examples. In Example 2 the sets $\mathcal{J}_{\mathcal{B}_{i}}\left(S_{i}\right), i=1,2$ will be empty and so no relation of the forms (V) and (VI) appears in the presentation, while in contrast in Example 3 we do get a relation of the form (V) (but again no relation of the form (VI)).

Example 1. Let $\mathcal{V}^{0}$ be a 4-dimensional real vector space with a basis $\mathcal{B}=\left\{\sigma_{1}, \sigma_{2}, \sigma_{3}, \sigma_{4}\right\}$. Let $\Lambda=\Sigma_{r=1}^{4} \mathbb{Z} \sigma_{r}$ and

$$
S=2 \Lambda \cup\left(\cup_{r=1}^{4}\left(\sigma_{r}+2 \Lambda\right)\right) \cup\left(\sigma_{1}+\sigma_{2}+2 \Lambda\right) \cup\left(\sigma_{1}+\sigma_{3}+\sigma_{4}+2 \Lambda\right) .
$$

Then $S$ is a semilattice in $\mathcal{V}^{0}$ of index 6 and $\mathcal{B}$ is a basis for $S$. Moreover,

$$
\operatorname{supp}_{\mathcal{B}}(S)=\{\emptyset,\{1\},\{2\},\{3\},\{4\},\{1,2\},\{1,3,4\}\} .
$$

According to (1.2) we have

$$
\begin{gathered}
\delta(1,2)=1, \\
\delta(1,3)=\delta(1,4)=\delta(2,3)=\delta(2,4)=\delta(3,4)=2, \\
\delta(3,1)=\delta(4,1)=\delta(3,2)=\delta(4,2)=\delta(4,3)=-2 \quad \text { and } \quad \delta(2,1)=-1 .
\end{gathered}
$$

Also we have $\mathcal{J}_{\mathcal{B}}(S)=\{\{1,3,4\}\}$.

Example 2. Let $\mathcal{V}=\Sigma_{i=1}^{2} \mathbb{R} \alpha_{i} \oplus \Sigma_{r=1}^{3} \mathbb{R} \sigma_{r}$ be a 5 -dimensional real vector space equipped with the positive semi-definite symmetric bilinear form $(\cdot, \cdot)$ given by

$$
\begin{aligned}
& \mathcal{V}^{0}:=\sum_{i=1}^{3} \mathbb{R} \sigma_{i} \text { is the radical of the form, } \\
& \left(\alpha_{1}, \alpha_{1}\right)=2, \quad\left(\alpha_{2}, \alpha_{2}\right)=4, \quad\left(\alpha_{1}, \alpha_{2}\right)=-2 .
\end{aligned}
$$

Let $\dot{R}$ be the finite root system in $\dot{\mathcal{V}}=\Sigma_{i=1}^{2} \mathbb{R} \alpha_{i}$ of type $X_{\ell}=B_{2}$ with basis $\dot{\Pi}=\left\{\alpha_{1}, \alpha_{2}\right\}$ and the Cartan matrix (relative to $\dot{\Pi}$ )

$$
A=\left(\begin{array}{ll}
a_{1,1} & a_{1,2} \\
a_{2,1} & a_{2,2}
\end{array}\right)=\left(\begin{array}{cc}
2 & -1 \\
-2 & 2
\end{array}\right) .
$$

Let $S$ be the semilattice in $\mathcal{V}^{0}$ with basis $\mathcal{B}=\left\{\sigma_{1}, \sigma_{2}, \sigma_{3}\right\}$ such that

$$
\operatorname{supp}_{\mathcal{B}_{1}}(S)=\{\emptyset,\{1\},\{2\},\{3\},\{1,2\},\{1,3\}\} \text {. }
$$

Then

$$
R:=(S+S) \cup\left(\dot{R}_{s h}+S\right) \cup\left(\dot{R}_{l g}+2\langle S\rangle\right)
$$


is an extended affine root system in $\mathcal{V}$ of type $X_{\ell}=B_{2}$, of nullity $\nu=3$ and of twist number $t=3$. Comparing (4.3) with (1.9) we have $S=S_{1}, S_{2}=0$ and $R=S\left(B_{2}, S_{1}, 0\right)$. Since $\alpha_{1} \in \dot{R}_{s h}$ and $\alpha_{2} \in \dot{R}_{l g}$, the integers $k_{r}$ 's and $k_{i, r}$ 's (relative to the basis $\dot{\Pi}=\left\{\alpha_{1}, \alpha_{2}\right\}$ of $\dot{R}$ ) introduced in (2.5) and (2.6) are given by

$$
k_{1}=k_{2}=k_{3}=k_{2,1}=k_{2,2}=k_{2,3}=2 \quad \text { and } \quad k_{1,1}=k_{1,2}=k_{1,3}=1
$$

and so from the way that $t_{i, r}$ 's and $\Delta(r, s)$ 's are defined (see (2.9) and (2.12)) we have

$$
t_{1, r}=w_{\alpha_{1}+\sigma_{r}} w_{\alpha_{1}} \quad \text { and } \quad t_{2, r}=w_{\alpha_{2}+2 \sigma_{r}} w_{\alpha_{2}} \quad(1 \leq r \leq 3)
$$

and

$$
\Delta(1,2)=\Delta(1,3)=1 \quad \text { and } \quad \Delta(2,3)=2 .
$$

Then using (2.13), (4.4) and (4.6) we have

$$
z_{\{1,2\}}=c_{1,2}^{2}, \quad z_{\{1,3\}}=c_{1,3}^{2} \quad \text { and } \quad z_{\{2,3\}}=c_{2,3}^{4} .
$$

Let $\mathcal{W}$ be the extended affine Weyl group of $R$. Then from Proposition 2.1(ii) and the fact that $\mathcal{J}_{\mathcal{B}_{1}}\left(S_{1}\right)=\mathcal{J}_{\mathcal{B}_{2}}\left(S_{2}\right)=\emptyset$, we see that $\mathcal{W}$ is generated by elements

$$
w_{\alpha_{1}}, w_{\alpha_{2}}, t_{1, r}, t_{2, r},(1 \leq r \leq 3) \quad \text { and } \quad z_{\{r, s\}},(1 \leq r<s \leq 3) .
$$

Next using (4.1) and (4.4) we have for $1 \leq r \leq 3$ and $1 \leq r<s \leq 3$ (see (2.16) and $(2.17))$

$$
\begin{gathered}
a_{1,1}(r, s)=2, a_{2,2}(r, s)=4, a_{1,2}(r, s)=-2, a_{2,1}(r, s)=-2, \\
a_{1,1}(r)=a_{2,2}(r)=2, a_{1,2}(r)=-2, a_{2,1}(r)=-1 .
\end{gathered}
$$

Therefore from (4.1), (4.2), (4.6), and Theorem 3.1, it follows that the extended affine Weyl group of $R$ is isomorphic to the group defined by generators

$$
\hat{w}_{1}, \hat{w}_{2}, \hat{t}_{1, r}, \hat{t}_{2, r},(1 \leq r \leq 3) \quad \text { and } \quad \hat{z}_{r, s},(1 \leq r<s \leq 3),
$$

and relations

$$
\begin{aligned}
& \hat{w}_{i}^{2}=1, \quad\left(\hat{w}_{1} \hat{w}_{2}\right)^{4}=1, \quad \hat{w}_{1} \hat{t}_{1, r} \hat{w}_{1}=\hat{t}_{1, r}^{-1}, \quad \hat{w}_{2} \hat{t}_{2, r} \hat{w}_{2}=\hat{t}_{2, r}^{-1}, \\
& \hat{w}_{1} \hat{t}_{2, r} \hat{w}_{1}=\hat{t}_{2, r} \hat{t}_{1, r}^{2}, \quad \hat{w}_{2} \hat{t}_{1, r} \hat{w}_{2}=\hat{t}_{1, r} \hat{t}_{2, r}, \\
& {\left[\hat{t}_{1,1}, \hat{t}_{1,2}\right]=\left[\hat{t}_{2,2}, \hat{t}_{1,1}\right]=\left[\hat{t}_{1,2}, \hat{t}_{2,1}\right]=\hat{z}_{1,2}^{2}, \quad\left[\hat{t}_{2,1}, \hat{t}_{2,2}\right]=\hat{z}_{1,2}^{4},} \\
& {\left[\hat{t}_{1,1}, \hat{t}_{1,3}\right]=\left[\hat{t}_{2,3}, \hat{t}_{1,1}\right]=\left[\hat{t}_{1,3}, \hat{t}_{2,1}\right]=\hat{z}_{1,3}^{2}, \quad\left[\hat{t}_{2,1}, \hat{t}_{2,3}\right]=\hat{z}_{1,3}^{4},} \\
& {\left[\hat{t}_{1,2}, \hat{t}_{1,3}\right]=\left[\hat{t}_{2,3}, \hat{t}_{1,2}\right]=\left[\hat{t}_{1,3}, \hat{t}_{2,2}\right]=\hat{z}_{2,3}, \quad\left[\hat{t}_{2,2}, \hat{t}_{2,3}\right]=\hat{z}_{2,3}^{2},}
\end{aligned}
$$
and

$\left[\hat{w}_{i}, \hat{z}_{r, s}\right]=\left[\hat{t}_{i, r}, \hat{z}_{r, s}\right]=\left[\hat{t}_{i, r}, \hat{t}_{j, r}\right]=\left[\hat{z}_{r, s}, \hat{z}_{r^{\prime}, s^{\prime}}\right]=1$. 
Example 3. Let $\mathcal{V}$ be a 9-dimensional real vector space with a fixed basis $\left\{\alpha_{1}, \alpha_{2}, \sigma_{1}, \ldots, \sigma_{7}\right\}$ equipped with the positive semi-definite symmetric bilinear form $(\cdot, \cdot)$ given by

$$
\begin{aligned}
& \mathcal{V}^{0}:=\sum_{i-1}^{7} \mathbb{R} \sigma_{i} \text { is the radical of the form, } \\
& \left(\alpha_{1}, \alpha_{1}\right)=2, \quad\left(\alpha_{2}, \alpha_{2}\right)=4, \quad\left(\alpha_{1}, \alpha_{2}\right)=-2 .
\end{aligned}
$$

Let $\dot{R}$ be the finite root system of type $B_{2}$ as in Example 2. Let $\mathcal{V}_{1}^{0}$ and $\mathcal{V}_{2}^{0}$ be the subspaces of $\mathcal{V}^{0}$ with bases $\mathcal{B}_{1}:=\left\{\sigma_{1}, \sigma_{2}, \sigma_{3}, \sigma_{4}\right\}$ and $\mathcal{B}_{2}:=\left\{\sigma_{5}, \sigma_{6}, \sigma_{7}\right\}$, respectively. Also, let $S_{1}$ and $S_{2}$ be the semilattices in $\mathcal{V}_{1}^{0}$ and $\mathcal{V}_{2}^{0}$ with supporting classes

$$
\operatorname{supp}_{\mathcal{B}_{1}}\left(S_{1}\right)=\{\emptyset,\{1\},\{2\},\{3\},\{4\},\{1,2\},\{1,3,4\}\}
$$

and

$$
\operatorname{supp}_{\mathcal{B}_{2}}\left(S_{2}\right)=\{\emptyset,\{5\},\{6\},\{7\},\{5,6\},\{5,7\}\} .
$$

Then

$$
\mathcal{J}_{\mathcal{B}_{1}}\left(S_{1}\right)=\{\{1,3,4\}\} \text { and } \mathcal{J}_{\mathcal{B}_{2}}\left(S_{2}\right)=\{\{\}\} \text {. }
$$

Now $S:=S_{1} \oplus\left\langle S_{2}\right\rangle$ is a semilattice in $\mathcal{\nu}^{0}$ and

$$
R:=R\left(B_{2}, S_{1}, S_{2}\right)=(S+S) \cup\left(\dot{R}_{s h}+S_{1} \oplus\left\langle S_{2}\right\rangle\right) \cup\left(\dot{R}_{l g}+2\left\langle S_{1}\right\rangle \oplus S_{2}\right)
$$

is an extended affine root system in $\mathcal{V}$ of type $X_{\ell}=B_{2}$, of nullity 7 and of twist number 4. Since $\dot{\Pi}=\left\{\alpha_{1}, \alpha_{2}\right\}$ is a basis for the finite root system $\dot{R}$ such that $\left(\alpha_{1}, \alpha_{2}\right) \in \dot{R}_{s h} \times \dot{R}_{l g}$, the integers $k_{r}$ 's, $k_{i, r}$ 's (relative to $\dot{\Pi}$ ) and $k\left(\alpha_{i}\right)$ 's, (see $(2.5)$ and (2.6)) are given by

$$
k_{1}=k_{2}=k_{3}=k_{4}=2, \quad k_{5}=k_{6}=k_{7}=1
$$

$$
k_{1, r}=1, k_{2, r}=k_{r}, \quad 1 \leq r \leq 7, \quad k\left(\alpha_{1}\right)=2 \text { and } k\left(\alpha_{2}\right)=1 .
$$

From (2.9) and (4.12) we have

$$
t_{1, r}=w_{\alpha_{1}+\sigma_{r}} w_{\alpha_{1}} \quad \text { and } \quad t_{2, r}=w_{\alpha_{2}+k_{r} \sigma_{r}} w_{\alpha_{2}} \quad(1 \leq r \leq 7) .
$$

Using (4.8) and (4.9) we obtain (see (2.12))

$$
\Delta(1,2)=\Delta(5,6)=\Delta(5,7)=\Delta(r, s)=1, \quad 1 \leq r \leq 4<s \leq 7
$$

and

(4.15) $\Delta(1,3)=\Delta(1,4)=\Delta(2,3)=\Delta(2,4)=\Delta(3,4)=\Delta(6,7)=2$. 
Then by (2.13) we have

$$
z_{\{r, s\}}=c_{r, s}^{\Delta(r, s) k_{r}}(1 \leq r<s \leq 7) \quad \text { and } \quad z_{\{1,3,4\}}=c_{1,3}^{2} c_{1,4}^{2} c_{3,4}^{2}
$$

where the integers $k_{r}$ 's and $\Delta(r, s)$ 's are given by (4.11) and (4.15). Finally, using (4.1), (2.16) and (2.17) we get (for all $1 \leq r \leq 7$ and $1 \leq r<s \leq 7$ )

$$
\begin{gathered}
a_{1,1}(r, s)=4 k_{r}^{-1}, \quad a_{2,2}(r, s)=2 k_{s}, \quad a_{1,2}(r, s)=-2 k_{r}^{-1} k_{s}, \\
a_{2,1}(r, s)=-2, \quad a_{1,1}(r)=a_{2,2}(r)=2, \quad a_{1,2}(r)=-k_{r}, \quad a_{2,1}(r)=-2 k_{r}^{-1}
\end{gathered}
$$

and so from Proposition 2.1 and Theorem 3.1, it follows that $\mathcal{W}$ is generated by elements

$$
w_{\alpha_{1}}, w_{\alpha_{2}}, t_{1, r}, t_{2, r},(1 \leq r \leq 7), z_{\{r, s\}},(1 \leq r<s) \leq 7, \quad \text { and } \quad z_{\{1,3,4\}} .
$$

Moreover $\mathcal{W}$ is isomorphic to the group defined by generators

$$
\hat{w}_{1}, \hat{w}_{2}, \hat{t}_{1, r}, \hat{t}_{2, r}(1 \leq r \leq 7), \hat{z}_{r, s}(1 \leq r<s \leq 7), \hat{z}
$$

and relations

$$
\begin{aligned}
& \hat{w}_{i}^{2}=1, \quad\left(\hat{w}_{1} \hat{w}_{2}\right)^{4}=1, \quad \hat{z}^{2}=\hat{z}_{1,3} \hat{z}_{1,4} \hat{z}_{3,4}, \quad \hat{w}_{1} \hat{t}_{1, r} \hat{w}_{1}=\hat{t}_{1, r}^{-1}, \\
& \hat{w}_{2} \hat{t}_{2, r} \hat{w}_{2}=\hat{t}_{2, r}^{-1}, \quad \hat{w}_{1} \hat{t}_{2, r} \hat{w}_{1}=\hat{t}_{2, r} \hat{t}_{1, r}^{k_{r}}, \quad \hat{w}_{2} \hat{t}_{1, r} \hat{w}_{2}=\hat{t}_{1, r} \hat{t}_{2, r}^{2 k_{r}^{-1}} \text {, } \\
& {\left[\hat{t}_{1, r}, \hat{t}_{1, s}\right]=\hat{z}_{r, s}^{4 k_{r}^{-1} \Delta(r, s)^{-1}}, \quad\left[\hat{t}_{2, r}, \hat{t}_{2, s}\right]=\hat{z}_{r, s}^{2 k_{s} \Delta(r, s)^{-1}}}
\end{aligned}
$$

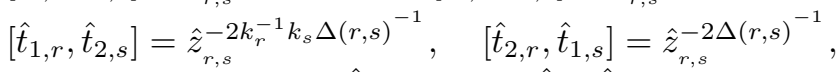

$$
\begin{aligned}
& {\left[\hat{w}_{i}, \hat{z}_{r, s}\right]=\left[\hat{w}_{i}, \hat{z}\right]=\left[\hat{t}_{i, r}, \hat{z}_{r, s}\right]=\left[\hat{t}_{i, r}, \hat{t}_{j, r}\right]=\left[\hat{z}_{r, s}, \hat{z}_{r^{\prime}, s^{\prime}}\right]=\left[\hat{z}_{r, s}, \hat{z}\right]=1,}
\end{aligned}
$$

where the integers $k_{r}$ 's and $\Delta(r, s)$ 's are given by (4.11), (4.14) and (4.15). We note that $\hat{z}^{2}=\hat{z}_{1,3} \hat{z}_{1,4} \hat{z}_{3,4}$ is the only relation of the form (V) which we get and that no relation of the form (VI) appears in this presentation as it is easy to see that for all $1 \leq r<s \leq 4, m_{r, s}=0$ are the only integers satisfying (2.24).

\section{References}

[AABGP] B. N. Allison, S. Azam, S. Berman, Y. Gao and A. Pianzola, Extended affine Lie algebras and their root systems, Mem. Amer. Math. Soc. 126 (1997), no. 603, $\mathrm{x}+122 \mathrm{pp}$.

[AzS1] S. Azam and V. Shahsanaei, Presentation by conjugation for $A_{1}$ types extended affine Weyl groups, J. Alg., to appear.

[AzS2] Simply laced extended affine Weyl groups (a finite presentation), Publ. Res. Inst. Math. Sci. 43 (2007), no. 2, 403-424.

[Az1] S. Azam, Extended affine Weyl groups, J. Algebra 214 (1999), no. 2, 571-624.

[Az2] A presentation for reduced extended affine Weyl groups, Comm. Algebra 28 (2000), no. 1, 465-488. 
[Az3] S. Azam, Extended affine root systems, J. Lie Theory 12 (2002), no. 2, 515-527.

[Az4] . Nonreduced extended affine Weyl groups, J. Algebra 269 (2003), no. 2, $508-527$.

[H] G. W. Hofmann, Weyl groups with Coxeter presentation and presentation by conjugation, J. Lie Theory 17 (2007), no. 2, 337-355.

[K] V. G. Kac, Infinite-dimensional Lie algebras, Third edition, Cambridge Univ. Press, Cambridge, 1990

[MS] R. V. Moody and Z. Shi, Toroidal Weyl groups, Nova J. Algebra Geom. 1 (1992), no. $4,317-337$.

[Sa] K. Saito, Extended affine root systems. I. Coxeter transformations, Publ. Res. Inst. Math. Sci. 21 (1985), no. 1, 75-179.

[SaT] K. Saito and T. Takebayashi, Extended affine root systems. III. Elliptic Weyl groups, Publ. Res. Inst. Math. Sci. 33 (1997), no. 2, 301-329.

[T1] T. Takebayashi, Defining relations of Weyl groups for extended affine root systems $A_{l}^{(1,1)}, B_{l}^{(1,1)}, C_{l}^{(1,1)}, D_{l}^{(1,1)}$, J. Algebra 168 (1994), no. 3, 810-827.

[T2] Weyl groups of the simply-laced 3-extended affine root systems, JP J. Algebra Number Theory Appl. 7 (2007), no. 1, 119-130. 